\title{
Double-critical graphs and complete minors
}

\author{
Ken-ichi Kawarabayashi \\ The National Institute of Informatics \\ 2-1-2 Hitotsubashi, Chiyoda-ku \\ Tokyo 101-8430, Japan \\ k_keniti@nii.ac.jp
}

\author{
Anders Sune Pedersen \& Bjarne Toft \\ Dept. of Mathematics and Computer Science \\ University of Southern Denmark \\ Campusvej 55, 5230 Odense M, Denmark \\ $\{$ asp, btoft\}@imada.sdu.dk
}

Submitted: Oct 14, 2008; Accepted: May 28, 2010; Published: Jun 7, 2010

Mathematics Subject Classification: 05C15, $05 \mathrm{C} 83$

\begin{abstract}
A connected $k$-chromatic graph $G$ is double-critical if for all edges $u v$ of $G$ the graph $G-u-v$ is $(k-2)$-colourable. The only known double-critical $k$-chromatic graph is the complete $k$-graph $K_{k}$. The conjecture that there are no other doublecritical graphs is a special case of a conjecture from 1966, due to Erdős and Lovász. The conjecture has been verified for $k$ at most 5 . We prove for $k=6$ and $k=7$ that any non-complete double-critical $k$-chromatic graph is 6 -connected and contains a complete $k$-graph as a minor.
\end{abstract}

\section{Introduction}

A long-standing conjecture, due to Erdős and Lovász [5], states that the complete graphs are the only double-critical graphs. We refer to this conjecture as the Double-Critical Graph Conjecture. A more elaborate statement of the conjecture is given in Section 2, where several other fundamental concepts used in the present paper are defined. The Double-Critical Graph Conjecture is easily seen to be true for double-critical $k$-chromatic graphs with $k$ at most 4. Mozhan [16] and Stiebitz [19, 20] independently proved the conjecture to hold for $k=5$, but it still remains open for all integers $k$ greater than 5 . The Double-Critical Graph Conjecture is a special case of a more general conjecture, the so-called Erdős-Lovász Tihany Conjecture [5], which states that for any graph $G$ with $\chi(G)>\omega(G)$ and any two integers $a, b \geqslant 2$ with $a+b=\chi(G)+1$, there is a partition $(A, B)$ of the vertex set $V(G)$ such that $\chi(G[A]) \geqslant a$ and $\chi(G[B]) \geqslant b$. The Erdös-Lovász Tihany Conjecture holds for every pair $(a, b) \in\{(2,2),(2,3),(2,4),(3,3),(3,4),(3,5)\}$ (see $[3,16,19,20])$. Kostochka and Stiebitz [13] proved it to be true for line graphs of multigraphs, while Balogh et al. [1] proved it to be true for quasi-line graphs and for graphs with independence number 2 . 
In addition, Stiebitz (private communication) has proved a weakening of the ErdősLovász Tihany conjecture, namely that for any graph $G$ with $\chi(G)>\omega(G)$ and any two integers $a, b \geqslant 2$ with $a+b=\chi(G)+1$, there are two disjoint subsets $A$ and $B$ of the vertex set $V(G)$ such that $\delta(G[A]) \geqslant a-1$ and $\delta(G[B]) \geqslant b-1$. (Note that for this conclusion to hold it is not enough to assume that $G \nsupseteq K_{a+b-1}$ and $\delta(G) \geqslant a+b-2$, that is, the Erdős-Lovász Tihany conjecture does not hold in general for the so-called colouring number. The 6-cycle with all shortest diagonals added is a counterexample with $a=2$ and $b=4$.) For $a=2$, the truth of this weaker version of the Erdős-Lovász Tihany conjecture follows easily from Theorem 3.1 of the present paper.

Given the difficulty in settling the Double-Critical Graph Conjecture we pose the following weaker conjecture:

Conjecture 1.1. Every double-critical $k$-chromatic graph is contractible to the complete $k$-graph.

Conjecture 1.1 is a weaker version of Hadwiger's Conjecture [9], which states that every $k$-chromatic graph is contractible to the complete $k$-graph. Hadwiger's Conjecture is one of the most fundamental conjectures of Graph Theory, much effort has gone into settling it, but it remains open for $k \geqslant 7$. For more information on Hadwiger's Conjecture and related problems we refer the reader to [11, 22].

In this paper we mainly devote attention to the double-critical 7-chromatic graphs. It seems that relatively little is known about 7-chromatic graphs. Jakobsen [10] proved that every 7 -chromatic graph has a $K_{7}$ with two edges missing as a minor. It is apparently not known whether every 7 -chromatic graph is contractible to $K_{7}$ with one edge missing. Kawarabayashi and Toft [12] proved that every 7-chromatic graph is contractible to $K_{7}$ or $K_{4,4}$.

The main result of this paper is that any double-critical 6- or 7-chromatic graph is contractible to the complete graph on six or seven vertices, respectively. These results are proved in Sections 6 and 7 using results of Györi [8] and Mader [15], but not the Four Colour Theorem. Krusenstjerna-Hafstrøm and Toft [14] proved that any double-critical $k$-chromatic non-complete graph is 5 -connected and $(k+1)$-edge-connected. In Section 5 , we extend that result by proving that any double-critical $k$-chromatic non-complete graph is 6-connected. In Section 3, we exhibit a number of basic properties of double-critical non-complete graphs. In particular, we observe that the minimum degree of any doublecritical non-complete $k$-chromatic graph $G$ is at least $k+1$ and that no two vertices of degree $k+1$ are adjacent in $G$, cf. Proposition 3.9 and Theorem 3.1. Gallai [7] also used the concept of decomposable graphs in the study of critical graphs. In Section 4, we use double-critical decomposable graphs to study the maximum ratio between the number of double-critical edges in a non-complete critical graph and the size of the graph, in particular, we prove that, for every non-complete 4-critical graph $G$, this ratio is at most $1 / 2$ and the maximum is attained if and only if $G$ is a wheel. Finally, in Section 8, we study two variations of the concept of double-criticalness, which we have termed doubleedge-criticalness and mixed-double-criticalness. It turns out to be straightforward to show that the only double-edge-critical graphs and mixed-double-critical graphs are the 
complete graphs.

\section{Notation}

All graphs considered in this paper are simple and finite. We let $n(G)$ and $m(G)$ denote the order and size of a graph $G$, respectively. The path, the cycle and the complete graph on $n$ vertices is denoted $P_{n}, C_{n}$ and $K_{n}$, respectively. The length of a path or a cycle is its number of edges. The set of integers $\{1,2, \ldots, k\}$ will be denoted $[k]$. Given two isomorphic graphs $G$ and $H$, we may (with a slight but common abuse of notation) write $G=H$. A $k$-colouring of a graph $G$ is a function $\varphi$ from the vertex set $V(G)$ of $G$ into a set $\mathcal{C}$ of cardinality $k$ so that $\varphi(u) \neq \varphi(v)$ for every edge $u v \in E(G)$, and a graph is $k$-colourable if it has a $k$-colouring. The elements of the set $\mathcal{C}$ are referred to as colours, and a vertex $v \in V(G)$ is said to be assigned the colour $\varphi(v)$ by $\varphi$. The set of vertices $S$ assigned the same colour $c \in \mathcal{C}$ is said to constitute the colour class $c$. The minimum integer $k$ for which a graph $G$ is $k$-colourable is called its chromatic number of $G$ and it is denoted $\chi(G)$. An independent set $S$ of $G$ is a set such that the induced graph $G[S]$ is edge-empty. The maximum integer $k$ for which there exists an independent set $S$ of $G$ of cardinality $k$ is the independence number of $G$ and is denoted $\alpha(G)$. A graph $H$ is a minor of a graph $G$ if $H$ can be obtained from $G$ by deleting edges and/or vertices and contracting edges. An $H$-minor of $G$ is a minor of $G$ isomorphic to $H$. Given a graph $G$ and a subset $U$ of $V(G)$ such that the induced graph $G[U]$ is connected, the graph obtained from $G$ by contracting $U$ into one vertex is denoted $G / U$, and the vertex of $G / U$ corresponding to the set $U$ of $G$ is denoted $v_{U}$. Let $\delta(G)$ denote the minimum degree of $G$. For a vertex $v$ of a graph $G$, the (open) neighbourhood of $v$ in $G$ is denoted $N_{G}(v)$ while $N_{G}[v]$ denotes the closed neighbourhood $N_{G}(v) \cup\{v\}$. Given two subsets $X$ and $Y$ of $V(G)$, we denote by $E[X, Y]$ the set of edges of $G$ with one end-vertex in $X$ and the other end-vertex in $Y$, and by $e(X, Y)$ their number. If $X=Y$, then we simply write $E(X)$ and $e(X)$ for $E[X, X]$ and $e(X, X)$, respectively. The induced graph $G[N(v)]$ is refered to as the neighbourhood graph of $v$ (w.r.t. $G$ ) and it is denoted $G_{v}$. The independence number $\alpha\left(G_{v}\right)$ is denoted $\alpha_{v}$. The degree of a vertex $v$ in $G$ is denoted $\operatorname{deg}_{G}(v)$ or $\operatorname{deg}(v)$. A graph $G$ is called vertex-critical or, simply, critical if $\chi(G-v)<\chi(G)$ for every vertex $v \in V(G)$. A connected graph $G$ is called double-critical if

$$
\chi(G-x-y) \leqslant \chi(G)-2 \text { for all edges } x y \in E(G)
$$

Of course, $\chi(G-x-y)$ can never be strictly less than $\chi(G)-2$, so we could require $\chi(G-x-y)=\chi(G)-2$ in (1). It is also clear that any double-critical graph is vertexcritical. The concept of vertex-critical graphs was first introduced by Dirac [4] and have since been studied extensively, see, for instance, [11]. As noted by Dirac [4], every critical $k$-chromatic graph $G$ has minimum degree $\delta(G) \geqslant k-1$. An edge $x y \in E(G)$ such that $\chi(G-x-y)=\chi(G)-2$ is referred to as a double-critical edge. For graph-theoretic terminology not explained in this paper, we refer the reader to [2]. 


\section{Basic properties of double-critical graphs}

In this section we let $G$ denote a non-complete double-critical $k$-chromatic graph. Thus, by the aforementioned results, $k$ is at least 6 .

Proposition 3.1. The graph $G$ does not contain a complete $(k-1)$-graph as a subgraph.

Proof. Suppose $G$ contains $K_{k-1}$ as a subgraph. Since $G$ is $k$-chromatic and doublecritical, it follows that $G-V\left(K_{k-1}\right)$ is edge-empty, but not vertex-empty. Since $G$ is also vertex-critical, $\delta(G) \geqslant k-1$, and therefore every $v \in V\left(G-K_{k-1}\right)$ is adjacent to every vertex of $V\left(K_{k-1}\right)$ in $G$, in particular, $G$ contains $K_{k}$ as a subgraph. Since $G$ is vertex-critical, $G=K_{k}$, a contradiction.

Proposition 3.2. If $H$ is a connected subgraph of $G$ with $n(H) \geqslant 2$, then the graph $G / V(H)$ obtained from $G$ by contracting $H$ is $(k-1)$-colourable.

Proof. The graph $H$ contains at least one edge $u v$, and the graph $G-u-v$ is $(k-2)$ colourable, which, in particular, implies that the graph $G-H$ is $(k-2)$-colourable. Now, any $(k-2)$-colouring of $G-H$ may be extended to a $(k-1)$-colouring of $G / V(H)$ by assigning a new colour to the vertex $v_{V(H)}$.

Given any edge $x y \in E(G)$, define

$$
\begin{aligned}
A(x y) & :=N(x) \backslash N[y] \\
B(x y) & :=N(x) \cap N(y) \\
C(x y) & :=N(y) \backslash N[x] \\
D(x y) & :=V(G) \backslash(N(x) \cup N(y)) \\
& =V(G) \backslash(A(x y) \cup B(x y) \cup C(x y) \cup\{x, y\})
\end{aligned}
$$

We refer to $B(x y)$ as the common neighbourhood of $x$ and $y$ (in $G$ ).

In the proof of Proposition 3.3 we use what has become known as generalized Kempe chains, cf. [17, 21]. Given a $k$-colouring $\varphi$ of a graph $H$, a vertex $x \in H$ and a permutation $\pi$ of the colours $1,2, \ldots, k$. Let $N_{1}$ denote the set of neighbours of $x$ of colour $\pi(\varphi(x))$, let $N_{2}$ denote the set of neighbours of $N_{1}$ of colour $\pi(\pi(\varphi(x)))$, let $N_{3}$ denote the set of neighbours of $N_{2}$ of colour $\pi^{3}(\varphi(x))$, etc. We call $N(x, \varphi, \pi)=\{x\} \cup N_{1} \cup N_{2} \cup \cdots a$ generalized Kempe chain from $x$ w.r.t. $\varphi$ and $\pi$. Changing the colour $\varphi(y)$ for all vertices $y \in N(x, \varphi, \pi)$ from $\varphi(y)$ to $\pi(\varphi(y))$ gives a new $k$-colouring of $H$.

Proposition 3.3. For all edges $x y \in E(G)$, $(k-2)$-colourings of $G-x-y$ and any non-empty sequence $j_{1}, j_{2}, \ldots, j_{i}$ of $i$ different colours from $[k-2]$, there is a path of order $i+2$ starting at $x$, ending at $y$ and with the $t^{\prime}$ 'th vertex after $x$ having colour $j_{t}$ for all $t \in[i]$. In particular, $x y$ is contained in at least $(k-2) ! /(k-2-i)$ ! cycles of length $i+2$.

Proof. Let $x y$ denote an arbitrary edge of $G$ and let $\varphi$ denote a $(k-2)$-colouring of $G-x-y$ which uses the colours of $[k-2]$. The function $\varphi$ is extended to a proper $(k-1)$ colouring of $G-x y$ by defining $\varphi(x)=\varphi(y)=k-1$. Let $\pi$ denote the cyclic permutation 
$\left(k-1, j_{1}, j_{2}, \ldots, j_{i}\right)$. If the generalized Kempe chain $N(x, \varphi, \pi)$ does not contain the vertex $y$, then by reassigning colours on the vertices of $N(x, \varphi, \pi)$ as described above, a $(k-1)$ colouring $\psi$ of $G-x y$ with $\psi(x) \neq k-1=\psi(y)$ is obtained, contradicting the fact that $G$ is $k$-chromatic. Thus, the generalized Kempe chain $N(x, \varphi, \pi)$ must contain the vertex $y$. Since $x$ and $y$ are the only vertices which are assigned the colour $k-1$ by $\varphi$, it follows that the induced graph $G[N(x, \varphi, \pi)]$ contains an $(x, y)$-path of order $i+2$ with vertices coloured consecutively $k-1, j_{1}, j_{2}, \ldots, j_{i}, k-1$. The last claim of the proposition follows from the fact there are $(k-2) ! /(k-2-i)$ ! ways of selecting and ordering $i$ elements from the set $[k-2]$.

Note that the number of cycles of a given length obtained in Proposition 3.3 is exactly the number of such cycles in the complete $k$-graph. Moreover, Proposition 3.3 immediately implies the following result.

Corollary 3.1. For all edges $x y \in E(G)$ and $(k-2)$-colourings of $G-x-y$, the set $B(x y)$ of common neighbours of $x$ and $y$ in $G$ contains vertices from every colour class $i \in[k-2]$, in particular, $|B(x y)| \geqslant k-2$, and $x y$ is contained in at least $k-2$ triangles.

Proposition 3.4. For all vertices $x \in V(G)$, the minimum degree in the induced graph of the neighbourhood of $x$ in $G$ is at least $k-2$, that is, $\delta\left(G_{x}\right) \geqslant k-2$.

Proof. According to Corollary 3.1, $|B(x y)| \geqslant k-2$ for any vertex $y \in N(x)$, which implies that $y$ has at least $k-2$ neighbours in $G_{x}$.

Proposition 3.5. For any vertex $x \in V(G)$, there exists a vertex $y \in N(x)$ such that the set $A(x y)$ is not empty.

Proof. Let $x$ denote any vertex of $G$, and let $z$ in $N(x)$. The common neighbourhood $B(x z)$ contains at least $k-2$ vertices, and so, since $K_{k-1}$ is not a subgraph of $G$, not every pair of vertices of $B(x y)$ are adjacent, say $y, y^{\prime} \in B(x z)$ are non-adjacent. Now $y^{\prime} \in A(x y)$, in particular, $A(x y)$ is not empty.

Proposition 3.6. There exists at least one edge $x y \in E(G)$ such that the set $D(x y)$ is not empty.

Proof. According to Proposition 3.5, there exists at least one edge $u v \in E(G)$ such that $A(u v)$ is not empty. Fix a vertex $a \in A(u v)$. This vertex $a$ cannot be adjacent to every vertex of $B(u v)$, since that, according to Corollary 3.1, would leave no colour available for $a$ in a $(k-2)$-colouring of $G-u-v$. Suppose $a$ is not adjacent to $z \in B(u v)$. Now $a \in D(v z)$, in particular, $D(v z)$ is not empty.

Proposition 3.7. If $A(x y)$ is not empty for some $x y \in E(G)$, then $\delta(G[A(x y)]) \geqslant 1$, that is, $G[A(x y)]$ contains no isolated vertices. By symmetry, $\delta(G[C(x y)]) \geqslant 1$, if $C(x y)$ is not empty. 
Proof. Suppose $G[A(x y)]$ contains some isolated vertex, say a. Now, since $G$ is doublecritical, $|B(x a)| \geqslant k-2$, and, since $a$ is isolated in $A(x y)$, the common neighbours of $x$ and $a$ must lie in $B(x y)$, in particular, any $(k-2)$-colouring of $G-a-x$ must assign all colours of the set $[k-2]$ to common neighbours of $a$ and $x$ in $B(x y)$. But this leaves no colour in the set $[k-2]$ available for $y$, which contradicts the fact that $G-a-x$ is $(k-2)$-colourable. This contradiction implies that $G[A(x y)]$ contains no isolated vertices.

Proposition 3.8. If some vertex $y \in N(x)$ is not adjacent to some vertex $z \in N(x) \backslash\{y\}$, then there exists another vertex $w \in N(x) \backslash\{y, z\}$, which is also not adjacent to $y$. Equivalently, no vertex of the complement $\overline{G_{x}}$ has degree 1 in $\overline{G_{x}}$.

Proof. The statement follows directly from Proposition 3.7. If $y \in N(x)$ is not adjacent to $z \in N(x) \backslash\{y\}$, then $z \in A(x y)$ and, since $G[A(x y)]$ contains no isolated vertices, the set $A(x y) \backslash\{z\}$ cannot be empty.

Proposition 3.9. Every vertex of $G$ has at least $k+1$ neighbours.

Proof. According to Proposition 3.5, for any vertex $x \in V(G)$, there exists a vertex $y \in N(x)$ such that $A(x y) \neq \emptyset$, and, according to Proposition 3.7, $\delta(G[A(x y)]) \geqslant 1$, in particular, $|A(x y)| \geqslant 2$. Since $N(x)$ is the union of the disjoint sets $A(x y), B(x y)$ and $\{y\}$, we obtain

$$
\operatorname{deg}_{G}(x)=|N(x)| \geqslant|A(x y)|+|B(x y)|+1 \geqslant 2+(k-2)+1=k+1
$$

where we used the fact that $|B(x y)| \geqslant k-2$, according to Corollary 3.1.

Proposition 3.10. For any vertex $x \in V(G)$,

$$
\operatorname{deg}_{G}(x)-\alpha_{x} \geqslant|B(x y)|+1 \geqslant k-1
$$

where $y \in N(x)$ is any vertex contained in an independent set in $N[x]$ of size $\alpha_{x}$. Moreover, $\alpha_{x} \geqslant 2$.

Proof. Let $S$ denote an independent set in $N(x)$ of size $\alpha_{x}$. Obviously, $\alpha_{x} \geqslant 2$, otherwise $G$ would contain a $K_{k}$. Choose some vertex $y \in S$. Now the non-empty set $S \backslash\{y\}$ is a subset of $A(x y)$, and, according to Proposition 3.7, $\delta(G[A(x y)]) \geqslant 1$. Let $a_{1}$ and $a_{2}$ denote two neighbouring vertices of $A(x y)$. The independet set $S$ of $G_{x}$ contains at most one of the vertices $a_{1}$ and $a_{2}$, say $a_{1} \notin S$. Therefore $S$ is a subset of $\{y\} \cup A(x y) \backslash\left\{a_{1}\right\}$, and so we obtain

$$
\alpha_{x} \leqslant|A(x y)|=|N(x)|-|B(x y)|-1 \leqslant \operatorname{deg}_{G}(x)-(k-2)-1
$$

from which (2) follows.

Proposition 3.11. For any vertex $x$ not adjacent to all other vertices of $G, \chi\left(G_{x}\right) \leqslant k-3$. 
Proof. Since $G$ is connected there must be some vertex, say $z$, in $V(G) \backslash N[x]$, which is adjacent to some vertex, say $y$, in $N(x)$. Now, clearly, $z$ is a vertex of $C(x y)$, in particular, $C(x y)$ is not empty, which, according to Proposition 3.7, implies that $C(x y)$ contains at least one edge, say $e=z v$. Since $G$ is double-critical, it follows that $\chi(G-z-v) \leqslant k-2$, in particular, the subgraph $G[N[x]]$ of $G-z-v$ is $(k-2)$-colourable, and so $G_{x}$ is $(k-3)$-colourable.

Proposition 3.12. If $\operatorname{deg}_{G}(x)=k+1$, then the complement $\overline{G_{x}}$ consists of isolated vertices (possibly none) and cycles (at least one), where the length of the cycles are at least five.

Proof. Given $\operatorname{deg}_{G}(x)=k+1$, suppose that some vertex $y \in G_{x}$ has three edges missing in $G_{x}$, say $y z_{1}, y z_{2}, y z_{3}$. Now $B(x y)$ is a subset of $N(x) \backslash\left\{y, z_{1}, z_{2}, z_{3}\right\}$. However, $\left|N(x) \backslash\left\{y, z_{1}, z_{2}, z_{3}\right\}\right|=(k+1)-4$, which implies $|B(x y)| \leqslant k-3$, contrary to Corollary 3.1. Thus no vertex of $G_{x}$ is missing more than two edges. According to Proposition 3.7, if a vertex of $G_{x}$ is missing one edge, then it is missing at least two edges. Thus, it follows that $\overline{G_{x}}$ consists of isolated vertices and cycles. If $\overline{G_{x}}$ consists of only isolated vertices, then $G_{x}$ would be a complete graph, and $G$ would contain a complete $(k+1)$-graph, contrary to our assumptions. Thus, $\overline{G_{x}}$ contains at least one cycle $C$. Let $s$ denote a vertex of $C$, and let $r$ and $t$ denote the two distinct vertices of $A(x s)$. Now $G-x-s$ is $(k-2)$-colourable and, according to Corollary 3.1, each of the $k-2$ colours is assigned to at least one vertex of the common neighbourhood $B(x s)$. Thus, both $r$ and $t$ must have at least one non-neighbour in $B(x s)$, and, since $r$ and $t$ are adjacent, it follows that $r$ and $t$ must have distinct non-neighbours, say $q$ and $u$, in $B(x s)$. Now, $q, r, s, t$ and $u$ induce a path of length four in $\overline{G_{x}}$ and so the cycle $C$ containing $P$ has length at least five.

Theorem 3.1. No two vertices of degree $k+1$ are adjacent in $G$.

Proof. Firstly, suppose $x$ and $y$ are two adjacent vertices of degree $k+1$ in $G$. Suppose that the one of the sets $A(x y)$ and $C(x y)$ is empty, say $A(x y)=\emptyset$. Then $|B(x y)|=k$ and $C(x y)=\emptyset$. Obviously, $\alpha_{x} \geqslant 2$, and it follows from Proposition 3.10 that $\alpha_{x}$ is equal to two. Let $\varphi$ denote a $(k-2)$-colouring of $G-x-y$. Now $|B(x y)|=k, \alpha_{x}=2$ and the fact that $\varphi$ applies each colour $c \in[k-2]$ to at least one vertex of $B(x y)$ implies that exactly two colours $i, j \in[k-2]$ are applied twice among the vertices of $B(x y)$, say $\varphi\left(u_{1}\right)=\varphi\left(u_{2}\right)=k-3$ and $\varphi\left(v_{1}\right)=\varphi\left(v_{2}\right)=k-2$, where $u_{1}, u_{2}, v_{1}$ and $v_{2}$ denotes four distinct vertices of $B(x y)$. Now each of the colours $1, \ldots, k-4$ appears exactly once in the colouring of the vertices of $W:=B(x y) \backslash\left\{u_{1}, u_{2}, v_{1}, v_{2}\right\}$, say $W=\left\{w_{1}, \ldots, w_{k-4}\right\}$ and $\varphi\left(w_{i}\right)=i$ for each $i \in[k-4]$. Now it follows from Proposition 3.3 that there exists a path $x w_{i} w_{j} y$ for each pair of distinct colours $i, j \in[k-4]$. Therefore $G[W]=K_{k-4}$. If one of the vertices $u_{1}, u_{2}, v_{1}$ or $v_{2}$, say $u_{1}$, is adjacent to every vertex of $W$, then $G\left[W \cup\left\{u_{1}, x, y\right\}\right]=K_{k-1}$, which contradicts Proposition 3.1. Hence each of the vertices $u_{1}, u_{2}, v_{1}$ and $v_{2}$ is missing at least one neighbour in $W$. It follows from Proposition 3.12, that the complement $\overline{G[B(x y)]}$ consists of isolated vertices and cycles of length at least five. Now it is easy to see that $\overline{G[B(x y)]}$ contains exactly one cycle, and we may w.l.o.g. assume 
that $u_{1} w_{1} v_{1} v_{2} w_{2} u_{2}$ are the vertices of that cycle. Now $G\left[\left\{u_{1}, v_{1}\right\} \cup W \backslash\left\{w_{1}\right\}\right]=K_{k-1}$, and we have again obtained a contradiction.

Secondly, suppose that one of the sets $A(x y)$ and $C(x y)$ is not empty, say $A(x y) \neq \emptyset$. Since, according to Corollary 3.1, the common neighbourhood $B(x y)$ contains at least $k-2$ vertices, it follows from Proposition 3.7 that $|A(x y)|=2$ and so $|B(x y)|=k-2$, which implies $|C(x y)|=2$. Suppose $A(x y)=\left\{a_{1}, a_{2}\right\}, C(x y)=\left\{c_{1}, c_{2}\right\}$, and let $C_{A}$ denote the cycle of the complement $\overline{G_{x}}$ which contains the vertices $a_{1}, y$ and $a_{2}$, say $C_{A}=a_{1} y a_{2} u_{1} \ldots u_{i}$, where $u_{1}, \ldots, u_{i} \in B(x y)$ and $i \geqslant 2$. Similarly, let $C_{C}$ denote the cycle of the complement $\overline{G_{y}}$ which contains the vertices $c_{1}, x$ and $c_{2}$, say $C_{A}=c_{1} x c_{2} v_{1} \ldots v_{j}$, where $v_{1}, \ldots, v_{j} \in B(x y)$ and $j \geqslant 2$. Since both $\overline{G_{x}}$ and $\overline{G_{y}}$ consists of only isolated vertices (possibly none) and cycles, it follows that we must have $\left(u_{1}, \ldots, u_{i}\right)=\left(v_{1}, \ldots, v_{j}\right)$ or $\left(u_{1}, \ldots, u_{i}\right)=\left(v_{j}, \ldots, v_{j}\right)$. We assume w.l.o.g. that the former holds.

Let $\varphi$ denote some $(k-2)$-colouring of $G-x-y$ using the colours of $[k-2]$, and suppose w.l.o.g. $\phi\left(a_{1}\right)=k-2$ and $\varphi\left(a_{2}\right)=k-3$. Again, the structure of $\overline{G_{x}}$ and $\overline{G_{y}}$ implies $\varphi\left(u_{1}\right)=k-3$ and $\varphi\left(u_{i}\right)=k-2$, which also implies $\varphi\left(c_{1}\right)=k-2$ and $\varphi\left(c_{2}\right)=k-3$.

Let $U=B(x y) \backslash\left\{u_{1}, u_{i}\right\}$. Now $U$ has size $k-4$ and precisely one vertex of $U$ is assigned the colour $i$ for each $i \in[k-4]$. Since no other vertices of $(N(x) \cup N(y)) \backslash U$ is assigned a colour from the set $[k-4]$, it follows from Proposition 3.3 that for each pair of distinct colours $s, t \in[k-4]$ there exists a path $x u^{s} u^{t} y$ where $u^{s}$ and $u^{t}$ are vertices of $U$ assigned the colours $s$ and $t$, respectively. This implies $G[U]=K_{k-4}$. No vertex of $G_{x}$ has more than two edges missing in $G_{x}$ and so, in particular, each of the adjacent vertices $a_{1}$ and $a_{2}$ are adjacent to every vertex of $U$. Now $G\left[U \cup\left\{a_{1}, a_{2}, x\right\}\right]=K_{k-1}$, which contradicts Proposition 3.1. Thus, no two vertices of degree $k+1$ are adjacent in $G$.

\section{Decomposable graphs and the ratio of double- critical edges in graphs}

A graph $G$ is called decomposable if it consists of two disjoint non-empty subgraphs $G_{1}$ and $G_{2}$ together with all edges joining a vertex of $G_{1}$ and a vertex of $G_{2}$.

Proposition 4.1. Let $G$ be a graph decomposable into $G_{1}$ and $G_{2}$. Then $G$ is doublecritical if and only if $G_{1}$ and $G_{2}$ are both double-critical.

Proof. Let $G$ be double-critical. Then $\chi(G)=\chi\left(G_{1}\right)+\chi\left(G_{2}\right)$. Moreover, for $x y \in E\left(G_{1}\right)$ we have

$$
\chi(G)-2=\chi(G-x-y)=\chi\left(G_{1}-x-y\right)+\chi\left(G_{2}\right)
$$

which implies $\chi\left(G_{1}-x-y\right)=\chi\left(G_{1}\right)-2$. Hence $G_{1}$ is double-critical, and similarly $G_{2}$ is.

Conversely, assume that $G_{1}$ and $G_{2}$ are both double-critical. Then for $x y \in E\left(G_{1}\right)$ we have

$$
\chi(G-x-y)=\chi\left(G_{1}-x-y\right)+\chi\left(G_{2}\right)=\chi\left(G_{1}\right)-2+\chi\left(G_{2}\right)=\chi(G)-2
$$


For $x y \in E\left(G_{2}\right)$ we have similarly that $\chi(G-x-y)=\chi(G)-2$. For $x \in V\left(G_{1}\right)$ and $y \in V\left(G_{2}\right)$ we have

$$
\chi(G-x-y)=\chi\left(G_{1}-x\right)+\chi\left(G_{2}-y\right)=\chi\left(G_{1}\right)-1+\chi\left(G_{2}\right)-1=\chi(G)-2
$$

Hence $\mathrm{G}$ is double-critical.

Gallai proved the theorem that a $k$-critical graph with at most $2 k-2$ vertices is always decomposable [6]. It follows easily from Gallai's Theorem, Proposition 4.1 and the fact that no double-critical non-complete graph with $\chi \leqslant 5$ exist, that a double-critical 6chromatic graph $G \neq K_{6}$ has at least 11 vertices. In fact, such a graph must have at least 12 vertices. Suppose $|V(G)|=11$. Then $G$ cannot be decomposable by Proposition 4.1; moreover, no vertex of a $k$-critical graph can have a vertex of degree $|V(G)|-2$; hence $\Delta(G)=8$ by Theorem 3.1, say $\operatorname{deg}(x)=8$. Let $y$ and $z$ denote the two vertices of $G-N[x]$. The vertices $y$ and $z$ have to be adjacent. Hence $\chi(G-y-z)=4$ and $\chi\left(G_{x}\right)=3$, which implies $\chi(G)=5$, a contradiction.

It also follows from Gallai's theorem and our results on double-critical 6- and 7chromatic graphs that any double-critical 8-chromatic graph without $K_{8}$ as a minor, if it exists, must have at least 15 vertices.

In the second part of the proof of Proposition 4.1, to prove that an edge $x y$ with $x \in V\left(G_{1}\right)$ and $y \in V\left(G_{2}\right)$ is double-critical in $G$, we only need that $x$ is critical in $G_{1}$ and $y$ is critical in $G_{2}$. Hence it is easy to find examples of critical graphs with many double-critical edges. Take for example two disjoint odd cycles of equal length $\geqslant 5$ and join them completely by edges. The result is a family of 6 -critical graphs in which the proportion of double-critical edges is as high as we want, say more than 99.99 percent of all edges may be double-critical. In general, for any integer $k \geqslant 6$, let $H_{k, \ell}$ denote the graph constructed by taking the complete $(k-6)$-graph and two copies of an odd cycle $C_{\ell}$ with $\ell \geqslant 5$ and joining these three graphs completely. Then the non-complete graph $H_{k, \ell}$ is $k$-critical, and the ratio of double-critical edges to the size of $H_{k, \ell}$ can be made arbitrarily close to 1 by choosing the integer $\ell$ sufficiently large. These observations perhaps indicate the difficulty in proving the Double-Critical Graph Conjecture: it is not enough to use just a few double-critical edges in a proof of the conjecture.

Taking an odd cycle $C_{\ell}(\ell \geqslant 5)$ and the complete 2-graph and joining them completely, we obtain a non-complete 5 -critical graph with at least $2 / 3$ of all edges being doublecritical. Maybe these graphs are best possible:

Conjecture 4.1. If $G$ denotes a 5-critical non-complete graph, then $G$ contains at most $c:=\left(2+\frac{1}{3 n(G)-5}\right) \frac{m(G)}{3}$ double-critical edges. Moreover, $G$ contains precisely $c$ doublecritical edges if and only if $G$ is decomposable into two graphs $G_{1}$ and $G_{2}$, where $G_{1}$ is the complete 2-graph and $G_{2}$ is an odd cycle of length $\geqslant 5$.

The conjecture, if true, would be an interesting extension of a theorem by Mozhan [16] and Stiebitz [20] which states that there is at least one non-double-critical edge. Computer tests using the list of vertex-critical graphs made available by Royle [18] indicate that Conjecture 4.1 holds for graphs of order less than 12. Moreover, the analogous statement 
holds for 4-critical graphs, cf. Theorem 4.1 below. In the proof of Theorem 4.1 we apply the following lemma, which is of interest in its own right.

Lemma 4.1. No non-complete 4-critical graph contains two non-incident double-critical edges.

Proof of Lemma 4.1. Suppose $G$ contains two non-incident double-critical edges $x y$ and $v w$. Since $\chi(G-\{v, w, x, y\})=2$, each component of $G-\{v, w, x, y\}$ is a bipartite graph. Let $A_{i}$ and $B_{i}(i \in[j])$ denote the partition sets of each bipartite component of $G-\{v, w, x, y\}$. (For each $i \in[j]$, at least one of the sets $A_{i}$ and $B_{i}$ are non-empty.) Since $G$ is critical, it follows that no clique of $G$ is a cut set of $G$ [2, Th. 14.7], in particular, both $G-x-y$ and $G-v-w$ are connected graphs. Hence, in $G-v-w$, there is at least one edge between a vertex of $\{x, y\}$ and a vertex of $A_{i} \cup B_{i}$ for each $i \in[j]$. Similarly, for $v$ and $w$ in $G-x-y$. If, say $x$ is adjacent to a vertex $a_{1} \in A_{i}$, then $y$ cannot be adjacent to a vertex $a_{2} \in A_{i}$, since then there would be a an even length $\left(a_{1}, a_{2}\right)$-path $P$ in the induced graph $G\left[A_{i} \cup B_{i}\right]$ and so the induced graph $G[V(P) \cup\{x, y\}]$ would contain an odd cycle, which contradicts the fact that the supergraph $G-v-w$ of $G[V(P) \cup\{x, y\}]$ is bipartite. Similarly, if $x$ is adjacent to a vertex of $A_{i}$, then $x$ cannot be adjacent to a vertex of $B_{i}$. Similar observations hold for $v$ and $w$. Let $A:=A_{1} \cup \cdots \cup A_{j}$ and $B:=B_{1} \cup \cdots \cup B_{j}$. We may w.l.o.g. assume that the neighbours of $x$ in $G-v-w-y$ are in the set $A$ and the neighbours of $y$ in $G-v-w-x$ are in $B$. In the following we distinguish between two cases.

(i) First, suppose that, in $G-x-y$, one of the vertices $v$ and $w$ is adjacent to only vertices of $A \cup\{v, w\}$, while the other is adjacent to only vertices of $B \cup\{v, w\}$. By symmetry, we may assume that $v$ in $G-x-y$ is adjacent to only vertices of $A \cup\{w\}$, while $w$ in $G-x-y$ is adjacent to only vertices of $B \cup\{v\}$. In this case we assign the colour 1 to the vertices of $A \cup\{w\}$, the colour 2 to the vertices of $B \cup\{v\}$.

Suppose that one of the edges $x v$ or $y w$ is not in $G$. By symmetry, it suffices to consider the case that $x v$ is not in $G$. In this case we assign the colour 2 to the vertex $x$ and the colour 3 to $y$. Since $x$ is not adjacent to any vertices of $B_{1} \cup \cdots \cup B_{j}$, we obtain a 3-colouring of $G$, which contradicts the assumption that $G$ is 4-chromatic.

Thus, both of the edges $x v$ and $y w$ are present in $G$. Suppose that $x w$ or $y v$ are missing from $G$. Again, by symmetry, it suffices to consider the case where yv is missing from $G$. Now assign the colour 2 to the vertex $x$ and the colour 3 to the vertex $y$ and a new colour to the vertex $v$. Again, we have a 3-colouring of $G$, a contradiction. Thus each of the edges $x w$ and $y v$ are in $G$, and so the vertices $x, y, v$ and $w$ induce a complete 4 -graph in $G$. However, no 4-critical graph $\neq K_{4}$ contains $K_{4}$ as a subgraph, and so we have a contradiction.

(ii) Suppose (i) is not the case. Then we may choose the notation such that there exist some integer $\ell \in\{2, \ldots, j\}$ such that for every integer $s \in\{1, \ldots, \ell\}$ the vertex $v$ is not adjacent to a vertex of $B_{s}$ and the vertex $w$ is not adjacent to a vertex of $A_{s}$; and for every integer $t \in\{\ell, \ldots, j\}$ the vertex $v$ is not adjacent to a vertex of $A_{t}$ and the vertex $w$ is not adjacent to a vertex of $B_{t}$. 
Since $G \nsubseteq K_{4}$, we may by symmetry assume that $x v \notin E(G)$. Now colour the vertices $v, x$ and all vertices of $B_{s}(s=1, \ldots, \ell-1)$ with colour 1 ; colour the vertex $w$, all vertices of $A_{s}(s=1, \ldots, \ell-1)$ and all vertices of $B_{t}(t=\ell, \ldots, j)$ with colour 2 ; and colour the vertex $y$ and all the vertices of $A_{t}(t=\ell, \ldots, j)$ with colour 3 . The result is a 3 -colouring of $G$. This contradicts $G$ being 4 -chromatic. Hence $G$ does not contain two non-incident double-critical edges.

Theorem 4.1. If $G$ denotes a 4-critical non-complete graph, then $G$ contains at most $m(G) / 2$ double-critical edges. Moreover, $G$ contains precisely $m(G) / 2$ double-critical edges if and only if $G$ contains a vertex $v$ of degree $n(G)-1$ such that the graph $G-v$ is an odd cycle of length $\geqslant 5$.

Proof. Let $G$ denote a 4-critical non-complete graph. According to Lemma 4.1, $G$ contains no two non-incident double-critical edges, that is, every two double-critical edges of $G$ are incident. Then, either the double-critical edges of $G$ all share a common end-vertex or they induce a triangle. In the later case $G$ contains strictly less that $m(G) / 2$ doublecritical edges, since $n(G) \geqslant 5$ and $m(G) \geqslant 3 n(G) / 2>6$. In the former case, let $v$ denote the common endvertex of the double-critical edges.

Now, the number of double-critical edges is at most $\operatorname{deg}(v)$, which is at most $n(G)-1$. Since $G$ is 4-critical, it follows that $G-v$ is connected and 3-chromatic. Hence $G-v$ is connected and contains an odd cycle, which implies $m(G-v) \geqslant n(G-v)$. Hence $m(G)=\operatorname{deg}(v)+m(G-v) \geqslant \operatorname{deg}(v)+n(G)-1 \geqslant 2 \operatorname{deg}(v)$, which implies the desired inequality. If the inequality is, in fact, an equality, then $\operatorname{deg}(v)=n(G)-1$ and $G$ is decomposable with $G-v$ an odd cycle of length $\geqslant 5$. The reverse implication is just a simple calculation.

\section{Connectivity of double-critical graphs}

Proposition 5.1. Suppose $G$ is a non-complete double-critical $k$-chromatic graph with $k \geqslant 6$. Then no minimal separating set of $G$ can be partitioned into two disjoint sets $A$ and $B$ such that the induced graphs $G[A]$ and $G[B]$ are edge-empty and complete, respectively.

Proof. Suppose that some minimal separating set $S$ of $G$ can be partitioned into disjoint sets $A$ and $B$ such that $G[A]$ and $G[B]$ are edge-empty and complete, respectively. We may assume that $A$ is non-empty. Let $H_{1}$ denote a component of $G-S$, and let $H_{2}:=G-\left(S \cup V\left(H_{1}\right)\right)$. Since $A$ is not empty, there is at least one vertex $x \in A$, and, by the minimality of the separating set $S$, this vertex $x$ has neighbours in both $V\left(H_{1}\right)$ and $V\left(H_{2}\right)$, say $x$ is adjacent to $y_{1} \in V\left(H_{1}\right)$ and $y_{2} \in V\left(H_{2}\right)$. Since $G$ is double-critical, the graph $G-x-y_{2}$ is $(k-2)$-colourable, in particular, there exists a $(k-2)$-colouring $\varphi_{1}$ of the subgraph $G_{1}:=G\left[V\left(H_{1}\right) \cup B\right]$. Similarly, there exists a $(k-2)$-colouring $\varphi_{2}$ of $G_{2}:=G\left[V\left(H_{2}\right) \cup B\right]$. The two graphs have precisely the vertices of $B$ in common, 
and the vertices of $B$ induce a complete graph in both $G_{1}$ and $G_{2}$. Thus, both $\varphi_{1}$ and $\varphi_{2}$ use exactly $|B|$ colours to colour the vertices of $B$, assigning each vertex a unique colour. By permuting the colours assigned by, say $\varphi_{2}$, to the vertices of $B$, we may assume $\varphi_{1}(b)=\varphi_{2}(b)$ for every vertex $b \in B$. Now $\varphi_{1}$ and $\varphi_{2}$ can be combined into a $(k-2)$-colouring $\varphi$ of $G-A$. This colouring $\varphi$ may be extended to a $(k-1)$-colouring of $G$ by assigning every vertex of the independent set $A$ the some new colour. This contradicts the fact that $G$ is $k$-chromatic, and so no minimal separating set $S$ as assumed can exist.

Krusenstjerna-Hafstrøm and Toft [14] states that any double-critical $k$-chromatic noncomplete graph is 5 -connected and $(k+1)$-edge-connected. In the following we prove that any double-critical $k$-chromatic non-complete graph is 6-connected.

Theorem 5.1. Every double-critical $k$-chromatic non-complete graph is 6-connected.

Proof. Suppose $G$ is a double-critical $k$-chromatic non-complete graph. Then, by the results mentioned in Section $1, k$ is at least 6 . Recall, that any double-critical graph, by definition, is connected. Thus, since $G$ is not complete, there exists some subset $U \subseteq V(G)$ such that $G-U$ is disconnected. Let $S$ denote a minimal separating set of $G$. We show $|S| \geqslant 6$. If $|S| \leqslant 3$, then $S$ can be partitioned into two disjoint subset $A$ and $B$ such that the induced graphs $G[A]$ and $G[B]$ are edge-empty and complete, respectively, and, thus, we have a contradiction by Proposition 5.1. Suppose $|S| \geqslant 4$, and let $H_{1}$ and $H_{2}$ denote disjoint non-empty subgraphs of $G-S$ such that $G-S=H_{1} \cup H_{2}$.

If $|S| \leqslant 5$, then each vertex $v$ of $V\left(H_{1}\right)$ has at most five neighbours in $S$ and so $v$ must have at least two neighbours in $V\left(H_{1}\right)$, since $\delta(G) \geqslant k+1 \geqslant 7$. In particular, there is at least one edge $u_{1} u_{2}$ in $H_{1}$, and so $G-u_{1}-u_{2}$ is $(k-2)$-colourable. This implies that the subgraph $G_{2}:=G-H_{1}$ of $G-u_{1}-u_{2}$ is $(k-2)$-colourable. Let $\varphi_{2}$ denote a $(k-2)$-colouring of $G_{2}$. A similar argument shows that $G_{1}:=G-H_{2}$ is $(k-2)$-colourable. Let $\varphi_{1}$ denote a $(k-2)$-colouring of $G_{1}$. If $\varphi_{1}$ or $\varphi_{2}$ applies just one colour to the vertices of $S$, then $S$ is an independent set of $G$, which contradicts Proposition 5.1. Thus, we may assume that both $\varphi_{1}$ and $\varphi_{2}$ applies at least two colours to the vertices of $S$. Let $\left|\varphi_{i}(S)\right|$ denote the number of colours applied by $\varphi_{i}(i=1,2)$ to the vertices of $S$. By symmetry, we may assume $\left|\varphi_{1}(S)\right| \geqslant\left|\varphi_{2}(S)\right| \geqslant 2$.

Moreover, if $\left|\varphi_{1}(S)\right|=\left|\varphi_{2}(S)\right|=|S|$, then, clearly, the colours applied by say $\varphi_{1}$ may be permuted such that $\varphi_{1}(s)=\varphi_{2}(s)$ for every $s \in S$ and so $\varphi_{1}$ and $\varphi_{2}$ may be combined into a $(k-2)$-coloring of $G$, a contradiction. Thus, $\left|\varphi_{1}(S)\right|=|S| \operatorname{implies}\left|\varphi_{2}(S)\right|<|S|$.

In general, we redefine the $(k-2)$-colourings $\varphi_{1}$ and $\varphi_{2}$ into $(k-1)$-colourings of $G_{1}$ and $G_{2}$, respectively, such that, after a suitable permutation of the colours of say $\varphi_{1}$, $\varphi_{1}(s)=\varphi_{2}(s)$ for every vertex $s \in S$. Hereafter a proper $(k-1)$-colouring of $G$ may be defined as $\varphi(v)=\varphi_{1}(v)$ for every $v \in V\left(G_{1}\right)$ and $\varphi(v)=\varphi_{2}(v)$ for every $v \in V(G) \backslash V\left(G_{1}\right)$, which contradicts the fact that $G$ is $k$-chromatic. In the following cases we only state the appropriate redefinition of $\varphi_{1}$ and $\varphi_{2}$.

Suppose that $|S|=4$, say $S=\left\{v_{1}, v_{2}, v_{3}, v_{4}\right\}$. We consider several cases depending on the values of $\left|\varphi_{1}(S)\right|$ and $\left|\varphi_{2}(S)\right|$. If $\left|\varphi_{i}(S)\right|=2$ for some $i \in\{1,2\}$, then $\varphi_{i}$ must apply both colours twice on vertices of $S$ (by Proposition 5.1). 
(1) Suppose that $\left|\varphi_{1}(S)\right|=4$.

(1.1) Suppose that $\left|\varphi_{2}(S)\right|=3$. In this case $\varphi_{2}$ uses the same colour at two vertices of $S$, say $\varphi_{2}\left(v_{1}\right)=\varphi_{2}\left(v_{2}\right)$. We simply redefine $\varphi_{2}$ such that $\varphi_{2}\left(v_{1}\right)=k-1$. Now both $\varphi_{1}$ and $\varphi_{2}$ applies four distinct colours to the vertices of $S$ and so they may be combined into a $(k-1)$-colouring of $G$, a contradiction.

(1.2) Suppose that $\left|\varphi_{2}(S)\right|=2$, say $\varphi_{2}\left(v_{1}\right)=\varphi_{2}\left(v_{2}\right)$ and $\varphi_{2}\left(v_{3}\right)=\varphi_{2}\left(v_{4}\right)$. This implies $v_{1} v_{2} \notin E(G)$, and so $\varphi_{1}$ may be redefined such that $\varphi_{1}\left(v_{1}\right)=\varphi_{1}\left(v_{2}\right)=k-1$. Moreover, $\varphi_{2}$ is redefined such that $\varphi_{2}\left(v_{4}\right)=k-1$.

(2) Suppose that $\left|\varphi_{1}(S)\right|=3$, say $\varphi_{1}\left(v_{1}\right)=1, \varphi_{1}\left(v_{2}\right)=2$ and $\varphi_{1}\left(v_{3}\right)=\varphi_{1}\left(v_{4}\right)=3$.

(2.1) Suppose that $\left|\varphi_{2}(S)\right|=3$, say $\varphi_{2}(x)=\varphi_{2}(y)$ for two distinct vertices $x, y \in S$. Redefine $\varphi_{1}$ and $\varphi_{2}$ such that $\varphi_{1}\left(v_{4}\right)=k-1$ and $\varphi_{2}(x)=k-1$.

(2.2) Suppose that $\left|\varphi_{2}(S)\right|=2$. If $\varphi_{2}\left(v_{1}\right)=\varphi_{2}\left(v_{2}\right)$ and $\varphi_{2}\left(v_{3}\right)=\varphi_{2}\left(v_{4}\right)$, then the desired $(k-1)$-colourings are obtained by redefining $\varphi_{2}$ such that $\varphi_{2}\left(v_{2}\right)=k-1$. If $\varphi_{2}\left(v_{2}\right)=\varphi_{2}\left(v_{3}\right)$ and $\varphi_{2}\left(v_{4}\right)=\varphi_{2}\left(v_{1}\right)$, then the desired $(k-1)$-colourings are obtained by redefining $\varphi_{2}$ such that $\varphi_{2}\left(v_{3}\right)=\varphi_{2}\left(v_{4}\right)=k-1$.

(3) Suppose that $\left|\varphi_{1}(S)\right|=2$. This implies $\left|\varphi_{2}(S)\right|=2$. We may, w.l.o.g., assume $\varphi_{1}\left(v_{1}\right)=\varphi_{1}\left(v_{2}\right)$ and $\varphi_{1}\left(v_{3}\right)=\varphi_{1}\left(v_{4}\right)$, in particular, $v_{1} v_{2} \notin E(G)$. If $\varphi_{2}\left(v_{1}\right)=\varphi_{2}\left(v_{2}\right)$ and $\varphi_{2}\left(v_{3}\right)=\varphi_{2}\left(v_{4}\right)$, then, obviously, $\varphi_{1}$ and $\varphi_{2}$ may be combined into a $(k-2)$ colouring of $G$, a contradiction. Thus, we may assume that $\varphi_{2}\left(v_{2}\right)=\varphi_{2}\left(v_{3}\right)$ and $\varphi_{2}\left(v_{4}\right)=\varphi_{2}\left(v_{1}\right)$. In this case we redefine both $\varphi_{1}$ and $\varphi_{2}$ such that $\varphi_{1}\left(v_{4}\right)=k-1$, and, since $v_{1} v_{2} \notin E(G), \varphi_{2}\left(v_{1}\right)=\varphi_{2}\left(v_{2}\right)=k-1$.

This completes the case $|S|=4$. Suppose $|S|=5$, say $S=\left\{v_{1}, v_{2}, v_{3}, v_{4}, v_{5}\right\}$. According to Proposition 5.1, neither $\varphi_{1}$ nor $\varphi_{2}$ uses the same colour for more than three vertices. Suppose that one of the colourings $\varphi_{1}$ or $\varphi_{2}$, say $\varphi_{2}$, applies the same colour to three vertices of $S$, say $\varphi_{2}\left(v_{3}\right)=\varphi_{2}\left(v_{4}\right)=\varphi_{2}\left(v_{5}\right)$. Now $\left\{v_{3}, v_{4}, v_{5}\right\}$ is an independent set. If (i) $\varphi_{1}\left(v_{1}\right)=\varphi_{1}\left(v_{2}\right)$ and $\varphi_{2}\left(v_{1}\right)=\varphi_{2}\left(v_{2}\right)$ or (ii) $\varphi_{1}\left(v_{1}\right) \neq \varphi_{1}\left(v_{2}\right)$ and $\varphi_{2}\left(v_{1}\right) \neq \varphi_{2}\left(v_{2}\right)$, then we redefine $\varphi_{1}$ such that $\varphi_{1}\left(v_{3}\right)=\varphi_{1}\left(v_{4}\right)=\varphi_{1}\left(v_{5}\right)=k-1$, and so $\varphi_{1}$ and $\varphi_{2}$ may, after a suitable permutation of the colours of say $\varphi_{1}$, be combined into a $(k-1)$ colouring of $G$. Otherwise, if $\varphi_{1}\left(v_{1}\right) \neq \varphi_{1}\left(v_{2}\right)$ and $\varphi_{2}\left(v_{1}\right)=\varphi_{2}\left(v_{2}\right)$, then we redefine both $\varphi_{1}$ and $\varphi_{2}$ such that $\varphi_{1}\left(v_{3}\right)=\varphi_{1}\left(v_{4}\right)=\varphi_{1}\left(v_{5}\right)=k-1$ and $\varphi_{2}\left(v_{2}\right)=k-1$. If $\varphi_{1}\left(v_{1}\right)=\varphi_{1}\left(v_{2}\right)$ and $\varphi_{2}\left(v_{1}\right) \neq \varphi_{2}\left(v_{2}\right)$, then we redefine both $\varphi_{1}$ and $\varphi_{2}$ such that $\varphi_{1}\left(v_{3}\right)=\varphi_{1}\left(v_{4}\right)=\varphi_{1}\left(v_{5}\right)=k-1$ and $\varphi_{2}\left(v_{1}\right)=\varphi_{2}\left(v_{2}\right)=k-1$. In both cases $\varphi_{1}$ and $\varphi_{2}$ may be combined into a $(k-1)$-colouring of $G$ Thus, we may assume that neither $\varphi_{1}$ nor $\varphi_{2}$ applies the same colour to three or more vertices of $S$, in particular, $\left|\varphi_{i}(S)\right| \geqslant 3$ for both $i \in\{1,2\}$. Again, we may assume $\left|\varphi_{1}(S)\right| \geqslant\left|\varphi_{2}(S)\right|$.

(a) Suppose that $\left|\varphi_{1}(S)\right|=5$.

(a.1) Suppose that $\left|\varphi_{2}(S)\right|=4$ with say $\varphi_{2}\left(v_{4}\right)=\varphi_{2}\left(v_{5}\right)$. In this case $v_{4} v_{5} \notin E(G)$ and so we redefine $\varphi_{1}$ such that $\varphi_{1}\left(v_{4}\right)=\varphi_{1}\left(v_{5}\right)=k-1$. 
(a.2) Suppose that $\left|\varphi_{2}(S)\right|=3$. Since $\varphi_{2}$ cannot assign the same colour to three or more vertices of $S$, we may assume $\varphi_{2}\left(v_{2}\right)=\varphi_{2}\left(v_{3}\right)$ and $\varphi_{2}\left(v_{4}\right)=\varphi_{2}\left(v_{5}\right)$. In this case $v_{4} v_{5} \notin E(G)$, and so we redefine $\varphi_{1}$ and $\varphi_{2}$ such that $\varphi_{1}\left(v_{4}\right)=\varphi_{1}\left(v_{5}\right)=k-1$ and $\varphi_{2}\left(v_{3}\right)=k-1$.

(b) Suppose $\left|\varphi_{1}(S)\right|=4$, say $\varphi_{1}\left(v_{4}\right)=\varphi_{1}\left(v_{5}\right)$.

(b.1) Suppose $\left|\varphi_{2}(S)\right|=4$ with $\varphi_{2}(x)=\varphi_{2}(y)$ for two distinct vertices $x, y \in S$. In this case we redefine $\varphi_{1}$ and $\varphi_{2}$ such that $\varphi_{1}\left(v_{5}\right)=k-1$ and $\varphi_{2}(y)=k-1$.

(b.2) Suppose $\left|\varphi_{2}(S)\right|=3$. In this case we distinguish between two subcases depending on the number of colours $\varphi_{2}$ applies to the vertices of the set $\left\{v_{1}, v_{2}, v_{3}\right\}$. As noted earlier, we must have $\left|\varphi_{2}\left(\left\{v_{1}, v_{2}, v_{3}\right\}\right)\right| \geqslant 2$. If $\left|\varphi_{2}\left(\left\{v_{1}, v_{2}, v_{3}\right\}\right)\right|=3$, then we redefine $\varphi_{2}$ such that $\varphi_{2}\left(v_{4}\right)=\varphi_{2}\left(v_{5}\right)=k-1$. Otherwise, if $\left|\varphi_{2}\left(\left\{v_{1}, v_{2}, v_{3}\right\}\right)\right|=2$ with say $\varphi_{2}\left(v_{2}\right)=\varphi_{2}\left(v_{3}\right)$. Now $v_{2} v_{3}, v_{4} v_{5} \notin E(G)$ and so we redefine $\varphi_{1}$ and $\varphi_{2}$ such that $\varphi_{1}\left(v_{2}\right)=\varphi_{1}\left(v_{3}\right)=k-1$ and $\varphi_{2}\left(v_{4}\right)=\varphi_{2}\left(v_{5}\right)=k-1$.

(c) Suppose that $\left|\varphi_{1}(S)\right|=3$, say $\varphi_{1}\left(v_{2}\right)=\varphi_{1}\left(v_{3}\right)$ and $\varphi_{1}\left(v_{4}\right)=\varphi_{1}\left(v_{5}\right)$. In this case we must have $\left|\varphi_{2}(S)\right|=3$. As noted earlier, $\varphi_{2}$ does not assign the same colour to three vertices of $S$, and so we may assume $\varphi_{2}$ applies the colours 1,2 and 3 to the vertices of $S$ and that only one vertex of $S$ is assigned the colour 1 while two pairs of vertices of given the colours 2 and 3, respectively. We distinguish between four subcases depending on which vertex of $S$ is assigned the colour 1 by $\varphi_{2}$ and and the number of colours $\varphi_{2}$ applies to the vertices of the two sets $\left\{v_{2}, v_{3}\right\}$ and $\left\{v_{4}, v_{5}\right\}$. We may assume $\left|\varphi_{2}\left(\left\{v_{2}, v_{3}\right\}\right)\right| \geqslant\left|\varphi_{2}\left(\left\{v_{4}, v_{5}\right\}\right)\right|$.

(c.1) If $\left|\varphi_{2}\left(\left\{v_{2}, v_{3}\right\}\right)\right|=\left|\varphi_{2}\left(\left\{v_{4}, v_{5}\right\}\right)\right|=1$, then, clearly, $\varphi_{1}$ and $\varphi_{2}$ may be combined into a $(k-2)$-colouring of $G$, a contradiction.

(c.2) Suppose $\left|\varphi_{2}\left(\left\{v_{2}, v_{3}\right\}\right)\right|=2,\left|\varphi_{2}\left(\left\{v_{4}, v_{5}\right\}\right)\right|=2$ and $\varphi_{2}\left(v_{1}\right)=1$. Suppose that $\varphi_{2}$ assigns the colour 2 to the two distinct vertices $x, y \in S \backslash\left\{v_{1}\right\}$. Now we redefine $\varphi_{1}$ and $\varphi_{2}$ such that $\varphi_{1}(x)=\varphi_{1}(y)=k-1$ and $\varphi_{2}(z)=k-1$ for some vertex $z \in S \backslash\left\{v_{1}, x, y\right\}$.

(c.3) Suppose $\left|\varphi_{2}\left(\left\{v_{2}, v_{3}\right\}\right)\right|=2,\left|\varphi_{2}\left(\left\{v_{4}, v_{5}\right\}\right)\right|=2$ and $\varphi_{2}\left(v_{1}\right) \neq 1$, say $\varphi_{2}\left(v_{5}\right)=1$. In this case there is a vertex $x \in\left\{v_{2}, v_{3}\right\}$ such that $\varphi_{2}(x)=\varphi_{2}\left(v_{4}\right)$. Now we redefine $\varphi_{1}$ and $\varphi_{2}$ such that $\varphi_{1}(x)=\varphi_{1}\left(v_{4}\right)=k-1$ and $\varphi_{2}\left(v_{1}\right)=k-1$.

(c.4) If $\left|\varphi_{2}\left(\left\{v_{2}, v_{3}\right\}\right)\right|=2$ and $\left|\varphi_{2}\left(\left\{v_{4}, v_{5}\right\}\right)\right|=1$, then we redefine the mapping $\varphi_{2}$ such that $\varphi_{2}\left(v_{2}\right)=\varphi_{2}\left(v_{3}\right)=k-1$. 


\section{Double-critical 6-chromatic graphs}

In this section we prove, without use of the Four Colour Theorem, that any double-critical 6-chromatic graph is contractible to $K_{6}$.

Theorem 6.1. Every double-critical 6-chromatic graph $G$ contains $K_{6}$ as a minor.

Proof. If $G$ is a the complete 6-graph, then we are done. Hence we may assume that $G$ is not the complete 6-graph. Now, according to Proposition 3.9, $\delta(G) \geqslant 7$. Firstly, suppose that $\delta(G) \geqslant 8$. Then $m(G)=\frac{1}{2} \sum_{v \in V(G)} \operatorname{deg}(v) \geqslant 4 n(G)>4 n(G)-9$. Györi [8] and Mader [15] proved that any graph $H$ with $n(H) \geqslant 6$ and $m(H) \geqslant 4 n(H)-9$ is contractible to $K_{6}$, which implies the desired result. Secondly, suppose that $G$ contains a vertex, say $x$, of degree 7 . Let $y_{i}(i \in[7])$ denote the neighbours of $x$. Now, according to Proposition 3.12, the complement of the induced subgraph $G_{x}$ consists of isolated vertices and cycles (at least one) of length at least five. Since $n\left(G_{x}\right)=7$, the complement $\overline{G_{x}}$ must contain exactly one cycle $C_{\ell}$. We consider three cases depending on the length of $C_{\ell}$. Suppose $C_{\ell}=\left\{y_{1}, y_{2}, \ldots, y_{\ell}\right\}$. If $\ell=5$, then $\left\{y_{1}, y_{3}, y_{6}, y_{7}\right\}$ induces a $K_{4}$, and so $\left\{y_{1}, y_{3}, y_{6}, y_{7}, x\right\}$ induces a $K_{5}$, which contradicts Proposition 3.1. If $\ell=6$, then $\left\{y_{1}, y_{3}, y_{5}, y_{7}, x\right\}$ induces a $K_{5}$; again, a contradiction. Finally, if $\ell=7$, then by contracting the edges $y_{2} y_{5}$ and $y_{4} y_{7}$ of $G_{x}$ into two distinct vertices a complete 5-graph is obtained, as is readily verified. Since, by definition, $x$ is adjacent to every vertex of $V\left(G_{x}\right)$, it follows that $G$ is contractible to $K_{6}$.

The proof of Theorem 6.1 implies the following result.

Corollary 6.1. Every double-critical 6-chromatic graph $G$ with $\delta(G)=7$ has the property that for every vertex $x \in V(G)$ with $\operatorname{deg}(x)=7$, the complement $\overline{G_{x}}$ is a 7 -cycle.

\section{Double-critical 7-chromatic graphs}

Let $G$ denote a double-critical non-complete 7-chromatic graph. Recall, that given a vertex $x \in V(G)$, we let $G_{x}$ denote the induced graph $G[N(x)]$ and $\alpha_{x}:=\alpha\left(G_{x}\right)$. The following corollary is a direct consequence of Proposition 3.11.

Corollary 7.1. For any vertex $x$ of $G$ not joined to all other vertices, $\chi\left(G_{x}\right) \leqslant 4$.

Proposition 7.1. For any vertex $x$ of $G$ of degree $9, \alpha_{x}=3$.

Proof. It follows from Proposition 3.10, that $\alpha_{x}$ is at most 3. Since $\chi\left(G_{x}\right) \cdot \alpha_{x} \geqslant n\left(G_{x}\right)=9$, it follows from Corollary 7.1, that $\alpha_{x} \geqslant 9 / \chi\left(G_{x}\right) \geqslant 9 / 4$, which implies $\alpha_{x} \geqslant 3$. Thus, $\alpha_{x}=3$.

Proposition 7.2. If $x$ is a vertex of degree 9 in $G$, then the complement $\overline{G_{x}}$ does not contain a $K_{4}^{-}$as a subgraph. 
Proof. Let $x$ denote a vertex of degree 9 in $G$. By Proposition 3.4, the minimum degree in $G_{x}$ is at least $k-2=5$. Suppose that the vertices $y_{1}, y_{2}, z_{1}, z_{2}$ are the vertices of a subgraph $K_{4}^{-}$in $\overline{G_{x}}$, that is, a 4-cycle with a diagonal edge $y_{1} y_{2}$. The graph $G-x-y_{1}$ is 5 colourable, and, according to Corollary 3.1, every one of the five colours occurs in $B\left(x y_{1}\right)$. None of the vertices $y_{2}, z_{1}$ or $z_{2}$ are in $B\left(x y_{1}\right)$, that is, $B\left(x y_{1}\right) \subseteq V\left(G_{x}\right) \backslash\left\{y_{1}, y_{2}, z_{1}, z_{2}\right\}$. Now the vertex $y_{2}$ is not adjacent to every vertex of $B\left(x y_{1}\right)$, since that would leave none of the five colours available for properly colouring $y_{2}$. Thus, in $G_{x}$ the vertex $y_{2}$ has at least four non-neighbours $\left(y_{1}, z_{1}, z_{2}\right.$ and, at least, one vertex from $\left.B\left(x y_{1}\right)\right)$. Since $n\left(G_{x}\right)=9$, we find that $y_{2}$ has at most $8-4$ neighbours in $N[x]$, and we have a contradiction.

Proposition 7.3. For any vertex $x$ of degree 9 in $G$, any vertex of an $\alpha\left(G_{x}\right)$-set has degree 5 in the neighbourhood graph $G_{x}$.

Proof. Let $x$ denote vertex of $G$ of degree 9 , and let $W=\left\{w_{1}, w_{2}, w_{3}\right\}$ denote any independent set in $G_{x}$. This vertices of $W$ all have degree at most 6 in $G_{x}$ and, by Proposition 3.4, at least 5. Suppose that, say, $w_{1} \in W$ has degree 6 . Now $B\left(x w_{2}\right)$ is a subset of $N\left(w_{1} ; G_{x}\right)$, $G-x-w_{2}$ is 5-colourable, and, according to Corollary 3.1, every one of the five colours occurs in $B\left(x y_{1}\right)$. This, however, leaves none of the five colours available for $w_{1}$, and we have a contradiction. It follows that any vertex of an independent set of three vertices in $G_{x}$ have degree 5 in $G_{x}$.

Proposition 7.4. If $G$ has a vertex $x$ of degree 9 , then

(i) the vertices of any $\alpha_{x}$-set $W=\left\{w_{1}, w_{2}, w_{3}\right\}$ all have degree 5 in $G_{x}$,

(ii) the vertices of $V\left(G_{x}\right)$ have degree 5,6 or 8 in $G_{x}$,

(iii) every vertex $w_{i}(i=1,2,3)$ has exactly one private non-neighbour w.r.t. $W$ in $G_{x}$, that is, there exist three distinct vertices in $G_{x}-W$, which we denote by $y_{1}, y_{2}$ and $y_{3}$, such that each $w_{i}(i=1,2,3)$ is adjacent to every vertex of $G_{x}-\left(W \cup y_{i}\right)$, and

(iv) each vertex $y_{i}$ has a neighbour and non-neighbour in $V\left(G_{x}\right) \backslash\left(W \cup\left\{y_{1}, y_{2}, y_{3}\right\}\right)$ (see Figure 1).

In the following, let $W:=\left\{w_{1}, w_{2}, w_{3}\right\}, Y:=\left\{y_{1}, y_{2}, y_{3}\right\}$ and $Z:=V\left(G_{x}\right) \backslash(W \cup Y)$. Note that the above corollary does not claim that each vertex $y_{i}$ has a private nonneighbour in $Z$ w.r.t. to $Y$.

Proof. Claim (i) follows from Proposition 7.1 and Proposition 7.3. According to Proposition $3.4, \delta\left(G_{x}\right) \geqslant 5$, and, obviously, $\Delta\left(G_{x}\right) \leqslant 8$, since $n\left(G_{x}\right)=9$. If some vertex $y \in G_{x}$ has degree strictly less than 8, then, according to Proposition 3.8, it has at least two non-neighbours in $G_{x}$, that is, $\operatorname{deg}\left(y, G_{x}\right) \leqslant 8-2$. This establishes (ii). As for the claim (iii), each vertex $w_{i}(i=1,2,3)$ has exactly five neighbours in $V\left(G_{x}\right) \backslash W$, which is a set of six vertices, and so $w_{i}$ has exactly one non-neigbour in $V\left(G_{x}\right) \backslash W$. Suppose say $w_{1}$ and $w_{2}$ have a common non-neighbour in $V\left(G_{x}\right) \backslash W$, say $u$. Now the vertices $w_{1}, w_{2}, w_{3}$ and $u$ induce a $K_{4}$ or $K_{4}^{-}$in the complement $\overline{G_{x}}$, which contradicts Propositions 7.2. 


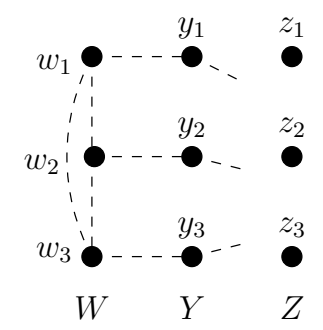

Figure 1: The graph $G_{x}$ as described in Proposition 7.4. The dashed curves indicate missing edges. The missing edges from $W$ to $Y \cup Z$ are exactly as indicated in the figure, while there may be more missing edges in $E\left(G_{x}-W\right)$ than indicated. The dashed curves starting at vertices of $y_{i}(i=1,2,3)$ and not ending at a vertex represent a missing edges between $y_{i}$ and a vertex of $Z$.

Hence, (iii) follows. Now for claim (iv). The fact that each vertex $y_{i}$ in $Y$ has at least one neighbour in $Z$ follows (ii) and the fact that $y_{i}$ is not adjacent to $w_{i}$. It remains to show that $y_{i}$ has at least one non-neighbour in $Z$. The graph $G-x-w_{1}$ is 5-colourable, in particular, there exists a 5-colouring $c$ of $G_{x}-w_{1}$, which, according to Corollary 3.1, assigns every colour from [5] to at least one vertex of $B\left(x w_{1}\right)$. In this case $B\left(x w_{1}\right)$ consists of precisely the vertices $y_{2}, y_{3}, z_{1}, z_{2}$ and $z_{3}$. We may assume $\varphi\left(y_{2}\right)=1, \varphi\left(y_{3}\right)=2$, $\varphi\left(z_{1}\right)=3, \varphi\left(z_{2}\right)=4$ and $\varphi\left(z_{3}\right)=5$. Since $w_{2}$ is adjacent to every vertex of $Z \cup Y \backslash\left\{y_{2}\right\}$, the only colour available for $w_{2}$ is the colour assign to $y_{2}$, that is, $\varphi\left(w_{2}\right)=\varphi\left(y_{2}\right)=1$. Similarly, $\varphi\left(w_{3}\right)=\varphi\left(y_{3}\right)=2$. Both the vertices $w_{2}$ and $w_{3}$ are adjacent to $y_{1}$ and so the colour assigned to $y_{1}$ cannot be one of the colours 1 or 2 , that is, $\varphi\left(y_{1}\right) \in\{3,4,5\}$. This implies, since $\varphi\left(z_{1}\right)=3, \varphi\left(z_{2}\right)=4$ and $\varphi\left(z_{3}\right)=5$, that $y_{1}$ cannot be adjacent to all three vertices $z_{1}, z_{2}$ and $z_{3}$. Thus, (iv) is established.

Corollary 7.2. If $G$ has a vertex $x$ of degree 9 , then there are at least two edges between vertices of $Y$.

Proof. If $m(G[Y]) \leqslant 1$, then it follows from (iiic) and (iv) of Proposition 7.4, that some vertex $y_{i} \in Y$ has at most four neighbours in $G_{x}$. But this contradicts (b) of the same proposition. Thus, $m(G[Y]) \geqslant 2$.

Lemma 7.1. If $x$ is a vertex of $G$ with minimum degree 9 and the neighbourhood graph $G_{x}$ is isomorphic to the graph $F$ of Figure 2, then $G$ is contractible to $K_{7}$.

Proof. According to Corollary 7.1, $\chi(G[N[x]]) \leqslant 5$, and so $N[x] \neq V(G)$. Let $H$ denote some component in $G-N[x]$. There are several ways of contracting $G_{x}$ to $K_{6}^{-}$. For instance, by contracting the three edges $w_{1} y_{3}, w_{2} y_{1}$ and $w_{3} y_{2}$ into three distinct vertices a $K_{6}^{-}$is obtained, where the vertices $z_{1}$ and $z_{3}$ remain non-adjacent. Thus, if there were a $z_{1}$ - $z_{3}$-path $P\left(z_{1}, z_{3}\right)$ with internal vertices completely contained in the set $V(G) \backslash N[x]$, then, by contracting the edges of $P\left(z_{1}, z_{3}\right)$, we would have a neighbourhood graph of $x$, which were contractible to $K_{6}$. Similarly, there exists contractions of $G_{x}$ such that if 


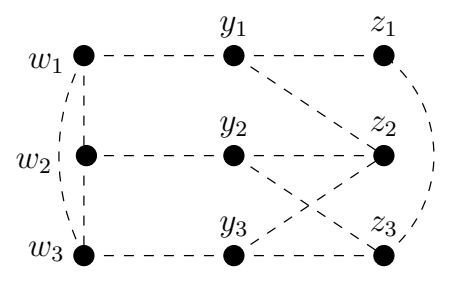

Figure 2: The graph $F$. The dashed lines between vertices indicate missing edges. Any edge which is not explicity indicated missing is present in $F$.

only there were a $w_{1}-y_{1}$-path $P\left(w_{1}, y_{1}\right), w_{2}$ - $y_{2}$-path $P\left(w_{2}, y_{1}\right)$ or $w_{3}$ - $y_{3}$-path $P\left(w_{3}, y_{3}\right)$ with internal vertices completely contained in the set $V(G) \backslash N[x]$, then such a path could be contracted such that the neighbourhood graph of $x$ would be contractible to $K_{6}$. Assume that none of the above mentioned paths $P\left(z_{1}, z_{3}\right), P\left(w_{1}, y_{1}\right), P\left(w_{2}, y_{1}\right)$ and $P\left(w_{3}, y_{3}\right)$ exist. In particular, for each pair of vertices $\left(z_{1}, z_{3}\right),\left(w_{1}, y_{1}\right),\left(w_{2}, y_{2}\right)$ and $\left(w_{3}, y_{3}\right)$ at most one vertex is adjacent to a vertex of $V(H)$, since if both, say $z_{1}$ and $z_{3}$ were adjacent to, say $u \in V(H)$ and $v \in V(H)$, respectively, then there would be a $z_{1}$ - $z_{3}$-path with internal vertices completely contained in the set $V(G) \backslash N[x]$, contradicting our assumption. Now it follows that in $G$ there can be at most five vertices of $V\left(G_{x}\right)$ adjacent to vertices of $V(H)$. By removing from $G$ the vertices of $V\left(G_{x}\right)$, which are adjacent to vertices of $V(H)$, the graph splits into at least two distinct components with $x$ in one component and the vertices of $V(H)$ in another component. This contradicts Theorem 5.1, which states that $G$ is 6-connected, and so the proof is complete.

Theorem 7.1. Every double-critical 7-chromatic graph $G$ contains $K_{7}$ as a minor.

Proof. If $G$ is a complete 7 -graph, then we are done. Hence, we may assume that $G$ is not a complete 7 -graph, and so, according to Proposition $3.9, \delta(G) \geqslant 8$. If $\delta(G) \geqslant 10$, then $m(G) \geqslant 5 n(G)>5 n-14$, and it follows from a theorem of Mader [15] that $G$ contains $K_{7}$ as a minor. Let $x$ denote a vertex of minimum degree. Suppose $\delta(G)=8$. Now, according to Proposition 3.12, the complement $\overline{G_{x}}$ consists of isolated vertices and cycles (at least one), each having length at least five. Since $n\left(G_{x}\right)=9$, it follows that $\overline{G_{x}}$ contains exactly one cycle $C_{\ell}$ of length at least 5 .

(i) If $\ell=5$, then $G\left[y_{1}, y_{3}, y_{6}, y_{7}, y_{8}, x\right]$ is the complete 6-graph, a contradiction.

(ii) If $\ell=6$, then $G\left[y_{1}, y_{3}, y_{5}, y_{7}, y_{8}, x\right]$ is the complete 6-graph, a contradiction.

(iii) If $\ell=7$, then by contracting the edges $y_{1} y_{4}$ and $y_{2} y_{6}$ of $G_{x}$ into two distinct vertices a complete 6 -graph is obtained, and so $G \geqslant K_{7}$.

(iv) If $\ell=8$, then by contracting the edges $y_{1} y_{5}$ and $y_{3} y_{7}$ of $G_{x}$ into two distinct vertices a complete 6 -graph is obtained, and so $G \geqslant K_{7}$.

Now, suppose $\delta(G)=9$. By Proposition 7.4, there is an $\alpha_{x}$-set $W=\left\{w_{1}, w_{2}, w_{3}\right\}$ of three distinct vertices such that there is a set $Y=\left\{y_{1}, y_{2}, y_{3}\right\} \subseteq V(G) \backslash W$ of three distinct 


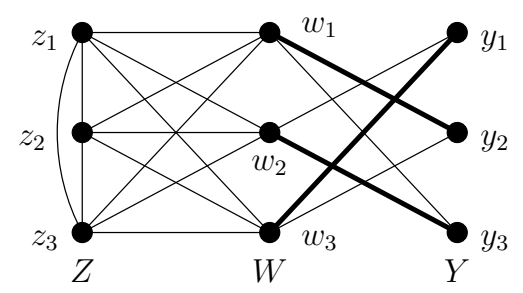

Figure 3: In Case 1.2.3, the graph $G_{x}$ contains the graph depicted above as a subgraph. The thick curves indicate the edges to be contracted. By contracting the three edges of $G_{x}$ as indicated above, a $K_{6}$ minor is obtained.

vertices such that $N\left(w_{i}, G_{x}\right)=V\left(G_{x}\right) \backslash\left(W \cup y_{i}\right)$ (see Figure 1). Let $Z=\left\{z_{1}, z_{2}, z_{3}\right\}$ denote the three remaining vertices of $G_{x}-(W \cup Y)$. We shall investigate the structure of $G_{x}$ and consider several cases. Thus, $e(W)=0$, and, as follows from Corollary 7.2, $e(Y) \geqslant 2$.

Suppose $e(Z)=3$. By contracting the edges $w_{1} y_{2}, w_{2} y_{3}$ and $w_{3} y_{1}$ of $G_{x}$ into three distinct vertices a complete 6 -graph is obtained (see Figure 3 ). Thus, $G \geqslant K_{7}$. In the following we shall be assuming $e(Z) \leqslant 2$.

Secondly, suppose $e(Z)=0$. Now $Z$ is an $\alpha_{x}$-set and it follows from Proposition 7.4, that $G_{x}$ possess the structure as indicated in Figure 4.

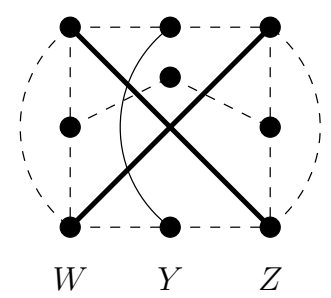

Figure 4: The graph $G_{x}$ contains the graph depicted above as a subgraph. The dashed curves represent edges missing in $G_{x}$. Except for the edges of $E(Y)$, any two pair of edge which are not explicity shown as non-adjacent are adjacent. The edge-set $E(Y)$ contains at least two edges. By symmetry, we assume $y_{1} y_{3} \in E(Y)$. By contracting two edges represented by thick curves, it becomes clear that $G_{x}$ contains $K_{6}$ as a minor.

By contracting the edges $w_{1} z_{3}$ and $w_{3} z_{1}$ of $G_{x}$ into two distinct vertices $w_{1}^{\prime}$ and $w_{3}^{\prime}$, we find that the vertices $w_{1}^{\prime}, w_{2}, w_{3}^{\prime}, y_{1}, y_{3}$ and $z_{2}$ induce a complete 6-graph, and we are done. Thus, in the following we shall be assuming $e(Z) \geqslant 1$. Moreover, we shall distinguish between several cases depending on the number of edges in $E(Y)$ and $E(Z)$. So far we have established $e(Y) \geqslant 2$ and $2 \geqslant e(Z) \geqslant 1$. We shall often use the fact that $\operatorname{deg}\left(u, G_{x}\right) \in\{5,6,8\}$ for every vertex $u \in G_{x}$, in particular, each vertex of $G_{x}$ can have at most three non-neighbours in $G_{x}$ (excluding itself).

(1) Suppose $e(Y)=3$. 


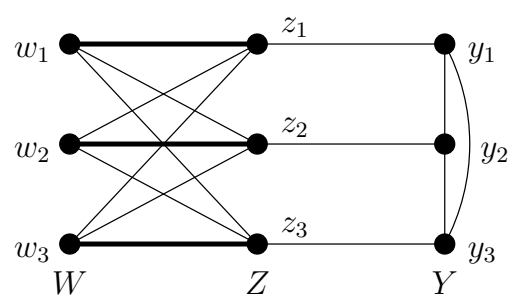

Figure 5: The graph $G_{x}$ contains the graph depicted above as a subgraph. The thick curves indicate the edges to be contracted. By contracting two edges of $G_{x}$ as indicated above, it becomes obvious that $G_{x}$ contains $K_{6}$ as a minor.

(1.1) If, in addition, there is a matching $M$ of $Y$ into $Z$, say $M=\left\{y_{1} z_{1}, y_{2} z_{2}, y_{3} z_{3}\right\}$, then contracting the edges $w_{i} z_{i}(i=1,2,3)$ into three distinct vertices results in a complete 6 -graph, and we are done (see Figure 5).

(1.2) Suppose that there is no matching of $Y$ into $Z$. Now it follows from Hall's Theorem [2, Th. 16.4] that there exists some non-empty set $S \subseteq Y$ such that $e(S, Z)<|S|$ (recall, that $e(S, Z)$ denotes the number of edges with one endvertex in $S$ and the other end-vertex in $Z$ ). According to Proposition 7.4, $e(S, Z) \geqslant 1$ for any non-empty $S \subseteq Y$.

(1.2.1) Suppose that $e(Y, Z)=1$, say $E(Y, Z)=\left\{z_{1}\right\}$. Now $y_{1}, y_{2}$ and $y_{3}$ are all nonneighbours of $z_{2}$ and $z_{3}$, and so both $z_{2}$ and $z_{3}$ must be adjacent to each other and to $z_{1}$, that is, $e(Z)=3$, contradicting our assumption that $e(Z) \leqslant 2$.

(1.2.2) Suppose that $e(Y, Z)=2$, say $E(Y, Z)=\left\{z_{1}, z_{2}\right\}$. Now $y_{1}, y_{2}$ and $y_{3}$ are three non-neighbours of $z_{3}$, and so $z_{3}$ must be adjacent to both $z_{2}$ and $z_{3}$. Since $e(Z) \leqslant 2$, it must be the case that $z_{1}$ and $z_{2}$ are non-neighbours. Since no vertex of $G_{x}$ has precisely one non-neighbour, both $z_{1}$ and $z_{2}$ must have at least one non-neighbour in $Y$. By symmetry, we may assume that $y_{1}$ is a non-neighbour of $z_{1}$. Now $w_{1}, z_{1}$ and $z_{3}$ are three non-neighbours of $y_{1}$, and so $y_{1}$ cannot be a non-neighbour of $z_{2}$. It follows that $y_{2}$ or $y_{3}$ must be a non-neighbour of $z_{2}$. By symmetry, we may assume $y_{2} z_{2} \notin E(G)$. Now there may be no more edges missing in $G_{x}$, however, we assume that there are more edges missing, and show that $G_{x}$ remains contractible to $K_{6}$. Each of the vertices $y_{1}$ and $y_{2}$ has three nonneighbours specified, while $y_{3}$ already has two non-neighbours specified. Thus, the only possible hitherto undetermined missing edge must be either $y_{3} z_{1}$ or $y_{3} z_{2}$ (not both, since that would imply $y_{3}$ to have at least four non-neighbours). By symmetry, we may assume $y_{3} z_{2} \notin E(G)$. Now it is clear that $G_{x}$ is isomorphic to the graph depicted in Figure 6, and so it follows from Lemma 7.1 that $G$ is contractible to $K_{7}$.

(1.2.3) Suppose that $e(Y, Z)=3$. Now, since there is no matching of $Y$ into $Z$ there must be some non-empty proper subset $S$ of $Y$ such that $|S| \leqslant 2$ and $e(S, Z)<$ 


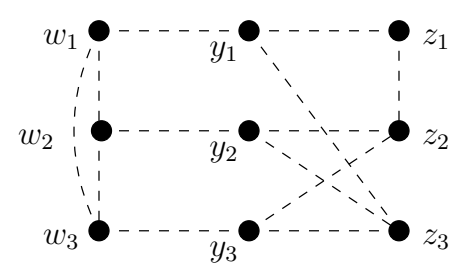

Figure 6: In Case 1.2.2, the graph $G_{x}$ is isomorphic to the graph depicted above. Any edge which is not explicity indicated missing is present.

$|S|$. Recall, $e(S, Z) \geqslant 1$ for any non-empty subset $S$ of $Y$, and so it must be the case that $|S|=2$ and $e(S, Z)=1$, say $S=\left\{y_{1}, y_{2}\right\}$ and $E(S, Z)=\left\{z_{1}\right\}$. The assumption $e(Y, Z)=3$ implies that $y_{3}$ is adjacent to both $z_{2}$ and $z_{3}$. According to Proposition 7.4 (iv), each vertex of $Y$ has a non-neighbour in $Z$, and so it must be the case that $y_{3}$ is not adjacent to $z_{1}$. Now, since $z_{1}$ has one nonneighbour in $V\left(G_{x}\right) \backslash\left\{z_{1}\right\}$, Proposition 3.8 (b) implies that it must have at least one other non-neighbour in $V\left(G_{x}\right)-z_{1}$. The only possible non-neighbours of $z_{1}$ in $V\left(G_{x}\right) \backslash\left\{z_{1}, y_{3}\right\}$ are $z_{2}$ and $z_{3}$, and, by symmetry, we may assume that $z_{1}$ and $z_{2}$ are not adjacent. Thus, $z_{2}$ is adjacent to neither $z_{1}, y_{1}$ nor $y_{2}$ and so $z_{2}$ must be adjacent to every vertex of $V\left(G_{x}\right) \backslash\left\{z_{1}, z_{2}, y_{1}, y_{2}\right\}$, in particular, $z_{2}$ is adjacent to $z_{3}$. Thus, $G_{x}$ contains the graph depicted in Figure 7 as a subgraph. Now, by contracting the edges $w_{1} z_{1}, w_{2} y_{1}$ and $w_{3} y_{2}$ of $G_{x}$ into three distinct vertices a complete 6-graph is obtained.

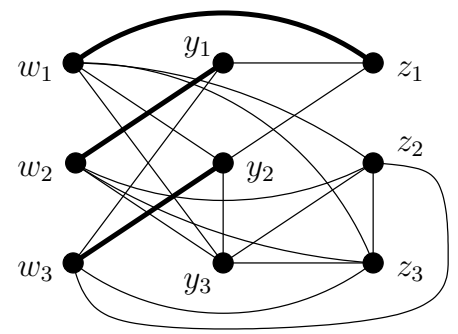

Figure 7: The graph $G_{x}$ contains the graph depicted above as a subgraph. The thick curves indicate the edges to be contracted. By contracting three edges of $G_{x}$ as indicated above, it becomes obvious that $G_{x}$ contains $K_{6}$ as a minor.

(2) Suppose $e(Y)=2$, say $y_{1} y_{2}, y_{2} y_{3} \in E(G)$.

(2.1) Suppose that $e(Z)=2$, say $z_{1} z_{2}, z_{2} z_{3} \in E(G)$.

(2.1.1) Suppose that at least one of the edges $y_{1} z_{1}$ or $y_{3} z_{3}$ are not in $E(G)$, say $y_{1} z_{1} \notin$ $E(G)$. The vertex $y_{1}$ has three non-neighbours in $G_{x}$, namely $w_{1}, y_{3}$ and $z_{1}$. Thus, $y_{1}$ must be adjacent to both $z_{2}$ and $z_{3}$. We have determined the edges 
of $E(W), E(Y)$ and $E(Z)$, and the edges joining vertices of $W$ with vertices of $Y \cup Z$. Moreover, $G_{x}$ contains at least two edges joining vertices of $Y$ with vertices of $Z$, as indicated in Figure 8 (a). It follows that $G_{x}$ contains the graph depicted in Figure 8 (b) as a subgraph. By contracting the edges $w_{1} y_{2}, w_{2} y_{3}$ and $w_{3} z_{1}$ of $G_{x}$ into three distinct vertices a complete 6-graph is obtained, and so $G \geqslant K_{7}$.

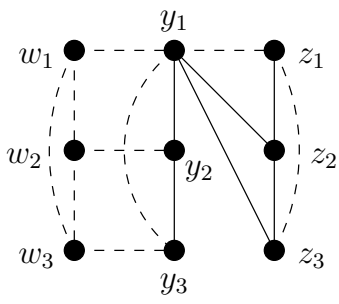

(a) The graph $G_{x}$ is completely determined, except for possible some edges between $Y$ and $Z$.

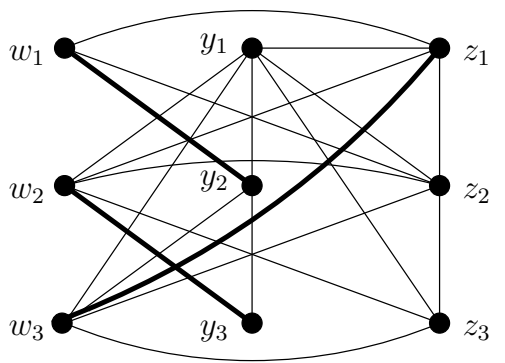

(b) The graph depicted above is a subgraph of $G_{x}$.

Figure 8: Illustration for Case 2.1.1.

(2.1.2) Suppose that both $y_{1} z_{1}$ and $y_{3} z_{3}$ are in $E(G)$.

(2.1.2.1) Suppose that $y_{1} z_{2}$ or $y_{3} z_{2}$ is in $E(G)$, say $y_{1} z_{2} \in E(G)$. In this case $G_{x}$ contains the graph depicted in Figure 9 (a) as a subgraph, and so by contracting the edges $w_{1} y_{2}, w_{2} y_{3}$ and $w_{3} z_{3}$ into three distinct vertices a complete 6-graph is obtained.

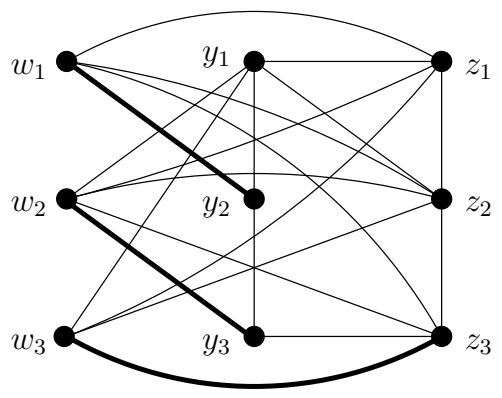

(a) In Case 2.1.2.1, $G_{x}$ contains the graph depicted above as a subgraph.

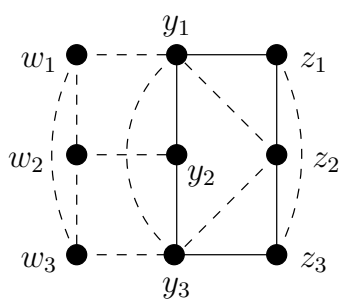

(b) In Case 2.1.2.2, $G_{x}$ is at least missing the edges as indicated in the above graph.

Figure 9: Illustration for Case 2.1.2. 
(2.1.2.2) Suppose that neither $y_{1} z_{2}$ nor $y_{3} z_{2}$ is in $E(G)$. Now $S:=\left\{y_{1}, z_{2}, y_{3}\right\}$ is an independent set of $G_{x}$ and so, according to Proposition 7.4 (iii), the vertex $z_{2}$ has a private non-neighbour in $V\left(G_{x}\right)-S$ w.r.t. $S$, and, as is easily seen from Figure 9 (b), the only possible non-neighbour of $z_{2}$ in $V\left(G_{x}\right)$ is $y_{2}$. The vertices $z_{1}$ and $z_{3}$ are not adjacent, and so, according to Proposition 7.4 (ii), each of them must have a second non-neighbour. Since $y_{1}$ and $y_{3}$ already have three non-neighbours specified, it follows that the only possible non-neighbour of $z_{1}$ and $z_{3}$ is $y_{2}$, but if neither $z_{1}$ nor $z_{3}$ are adjacent to $y_{2}$, then $y_{2}$ would have at least four non-neighbours in $G_{x}$, a contradiction.

(2.2) Suppose that $e(Z)=1$, say $E(Z)=\left\{z_{1} z_{3}\right\}$.

(2.2.1) Suppose that $y_{2} z_{2} \in E(G)$. Now at least one of the edges $y_{1} z_{2}$ and $y_{3} z_{2}$ is in $E(G)$, since otherwise $z_{2}$ would have at least four non-neighbour. By symmetry, we may assume $y_{1} z_{2} \in E(G)$. At least one of the edges $y_{1} z_{1}$ and $y_{1} z_{3}$ must be in $E(G)$, since $y_{1}$ cannot have more than three non-neighbours. By symmetry, we may assume $y_{1} z_{1} \in E(G)$ (see Figure 10 (a)). By contracting the edges $w_{1} z_{1}$, $w_{3} z_{3}$ and $y_{2} y_{3}$ of $G_{x}$ into three distinct vertices we obtain a complete 6-graph (see Figure $10(\mathrm{~b})$ ), and, thus, $G \geqslant K_{7}$.

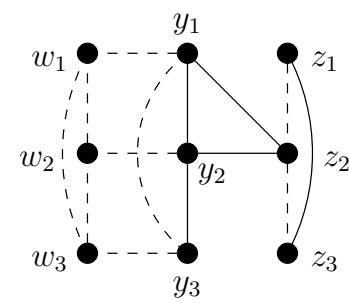

(a) The graph $G_{x}$ is completely determined, except for some edges between $Y$ and $Z$.

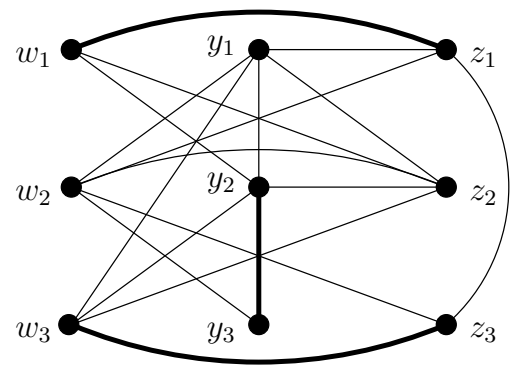

(b) The above graph is a subgraph of $G_{x}$.

Figure 10: Illustration for Case 2.2.1.

(2.2.2) Suppose that $y_{2} z_{2} \notin E(G)$. Each of the vertices $z_{1}$ and $z_{3}$ has exactly one nonneighbour in $Z$, namely $z_{2}$, and so each must have at least one non-neighbour in $Y$. If neither $z_{1}$ nor $z_{3}$ were adjacent to $y_{2}$, then $y_{2}$ would have at least four non-neighbours in $G_{x}$. Thus, at least one of $z_{1}$ and $z_{3}$ is not adjacent to $y_{1}$ or $y_{3}$. By symmetry, we may assume that $y_{1} z_{1} \notin E(G)$. Now we need to determine the non-neighbour of $y_{3}$ in $Y$.

(2.2.2.1) Suppose that $y_{2} z_{3} \in E(G)$. Since $y_{1}$ already has three non-neighbours, it must be the case that $y_{3}$ is a non-neighbour of $z_{3}$ in $Y$. There may also be an edge joining $y_{2}$ and $z_{1}$, but in any case $G_{x}$ contains the graph depicted in Figure 11 (a) 
as a subgraph. Thus, by contracting the edges $w_{2} z_{1}, w_{3} z_{1}$ and $y_{1} z_{2}$ into three distinct vertices, we find that $K_{6} \leqslant G_{x}$.

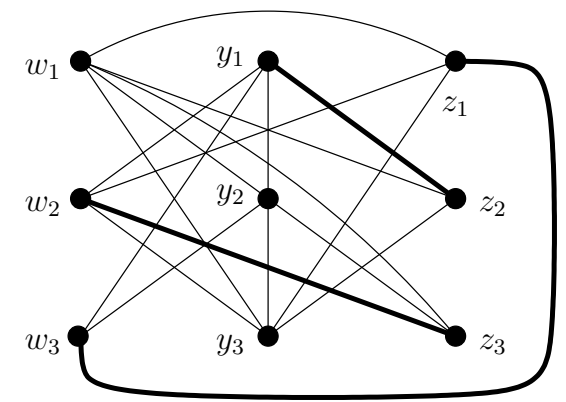

(a) In Case 2.2.2.1, $G_{x}$ contains the graph depicted above as a subgraph.

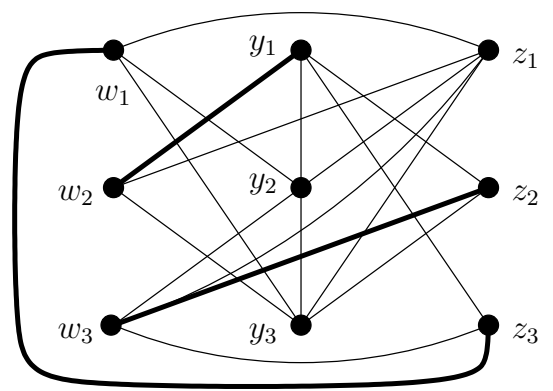

(b) In Case 2.2.2.2, $G_{x}$ contains the graph depicted above as a subgraph.

Figure 11: Illustration for Case 2.2.2.

(2.2.2.2) Suppose that $y_{2} z_{3} \notin E(G)$. In this case we find that $S:=\left\{y_{2}, z_{2}, z_{3}\right\}$ is a maximum independent set in $G_{x}$ and so, according to Proposition 7.4 (iii), each of the vertices of $S$ has a private non-neighbour in $V\left(G_{x}\right)-S$ w.r.t. $S$. The vertices $w_{1}, y_{3}$ and $z_{1}$ are all non-neighbours of $y_{1}$, and so $z_{3}$ cannot be a nonneighbour of $y_{1}$. It follows that the non-neighbour of $z_{3}$ in $V\left(G_{x}\right)-S$ must be $y_{3}$. Now each of the vertices of $Y$ has three non-neighbours, and so there can be no further edges missing from $G_{x}$, that is, $G_{x}$ contains the graph depicted in Figure 11 (b) as a subgraph.

This, finally, completes the case $\delta(G)=9$, and so the proof is complete.

Obviously, if every $k$-chromatic graph for some fixed integer $k$ is contractible to the complete $k$-graph, then every $\ell$-chromatic graph with $\ell \geqslant k$ is contractible to the complete $k$-graph. The corresponding result for double-critical graphs is not obviously true. However, for $k \leqslant 7$, it follows from the aforementioned results and Corollary 7.3 that every double-critical $\ell$-chromatic graph with $\ell \geqslant k$ is contractible to the complete $k$-graph.

Corollary 7.3. Every double-critical $k$-chromatic graph with $k \geqslant 7$ contains $K_{7}$ as a minor.

Proof. Let $G$ denote an arbitray double-critical $k$-chromatic graph with $k \geqslant 7$. If $G$ is complete, then we are done. If $k=7$, then the desired result follows from Theorem 7.1. If $k \geqslant 9$, then, according to Proposition 3.9, $\delta(G) \geqslant 10$ and so the desired result follows from a theorem of Györi [8] and Mader [15]. Suppose $k=8$ and that $G$ is non-complete. Then $\delta(G) \geqslant 9$. If $\delta(G) \geqslant 10$, then we are done and so we may assume $\delta(G)=9$, say $\operatorname{deg}(x)=9$. In this case it follows from Proposition 3.12 that the complement $\overline{G_{x}}$ consists of cycles (at least one) and isolated vertices (possibly none). An argument similar to the argument given in the proof of Theorem 6.1 shows that $G_{x}$ is contractible to $K_{6}$. Since $x$ dominates every vertex of $V\left(G_{x}\right)$, then $G$ itself is contractible to $K_{7}$. 
The problem of proving that every double-critical 8-chromatic graph is contractible to $K_{8}$ remains open.

\section{Double-edge-critical graphs and mixed-double- critical graphs}

A natural variation on the theme of double-critical graphs is to consider double-edgecritical graphs. A vertex-critical graph $G$ is called double-edge-critical if the chromatic number of $G$ decreases by at least two whenever two non-incident edges are removed from $G$, that is,

$$
\chi\left(G-e_{1}-e_{2}\right) \leqslant \chi(G)-2 \text { for any two non-incident edge } e_{1}, e_{2} \in E(G)
$$

It is easily seen that $\chi\left(G-e_{1}-e_{2}\right)$ can never be strictly less that $\chi(G)-2$ and so we may require $\chi\left(G-e_{1}-e_{2}\right)=\chi(G)-2$ in (3). The only critical $k$-chromatic graphs for $k \in\{1,2\}$ are $K_{1}$ and $K_{2}$, therefore we assume $k \geqslant 3$ in the following.

Theorem 8.1. A graph $G$ is $k$-chromatic double-edge-critical if and only if it is the complete k-graph.

Proof. It is straightforward to verify that any complete graph is double-edge-critical. Conversely, suppose $G$ is a $k$-chromatic $(k \geqslant 3)$ double-edge-critical graph. Then $G$ is connected. If $G$ is a complete graph, then we are done. Suppose $G$ is not a complete graph. Then $G$ contains an induced 3-path $P: w x y$. Since $G$ is vertex-critical, $\delta(G) \geqslant k-1 \geqslant 2$, and so $y$ is adjacent to some vertex $z$ is $V(G) \backslash\{w, x, y\}$. Now the edges $w x$ and $y z$ are not incident, and so $\chi(G-w x-y z)=k-2$. Let $\varphi$ denote a $(k-2)$-colouring of $G-w x-y z$. Then the vertices $w$ and $x$ (and $y$ and $z$ ) are assigned the same colours, since otherwise $G$ would be $(k-1)$-colourable. We may assume that $\varphi$ assigns the colour $k-3$ to the vertices $w$ and $x$, and the colour $k-2$ to the vertices $y$ and $z$. Now define the $(k-1)$-colouring $\varphi^{\prime}$ such that $\varphi^{\prime}(v)=\varphi(v)$ except $\varphi^{\prime}(w)=k-1$ and $\varphi^{\prime}(y)=k-1$. The colouring $\varphi^{\prime}$ is a proper $(k-1)$-colouring, since $w$ and $y$ are non-adjacent in $G$. This contradicts the fact that $G$ is $k$-chromatic and therefore $G$ must be a complete graph.

A vertex-critical $k$-chromatic graph $G$ is called mixed-double-critical if for any vertex $x \in G$ and any edge $e=u v \in E(G-x)$,

$$
\chi(G-x-e) \leqslant \chi(G)-2
$$

Theorem 8.2. A graph $G$ is k-chromatic mixed-double-critical if and only if it is the complete k-graph.

The proof of Theorem 8.2 is straightforward and similar to the proof of Theorem 8.1. 


\section{Acknowledgment}

The authors wish to thank Marco Chiarandini and Steffen Elberg Godskesen for creating computer programs for testing small graphs.

\section{References}

[1] J. Balogh, A. V. Kostochka, N. Prince, and M. Stiebitz. The Erdős-Lovász Tihany conjecture for quasi-line graphs. Discrete Math., 309(12):3985-3991, 2009.

[2] J. A. Bondy and U. S. R. Murty. Graph Theory, volume 244 of Graduate Texts in Mathematics. Springer, New York, 2008.

[3] W. G. Brown and H. A. Jung. On odd circuits in chromatic graphs. Acta Math. Acad. Sci. Hungar., 20:129-134, 1969.

[4] G. A. Dirac. A property of 4-chromatic graphs and some remarks on critical graphs. J. London Math. Soc., 27:85-92, 1952.

[5] P. Erdős. Problem 2. In Theory of Graphs (Proc. Colloq., Tihany, 1966), page 361. Academic Press, New York, 1968.

[6] T. Gallai. Critical graphs. In Theory of Graphs and its Applications (Proc. Sympos. Smolenice, 1963), pages 43-45. Publ. House Czechoslovak Acad. Sci., Prague, 1964.

[7] T. Gallai. Kritische Graphen. II. Magyar Tud. Akad. Mat. Kutató Int. Közl., 8: 373-395 (1964), 1963.

[8] E. Györi. On the edge numbers of graphs with Hadwiger number 4 and 5. Period. Math. Hungar., 13(1):21-27, 1982.

[9] H. Hadwiger. Über eine Klassifikation der Streckenkomplexe. Vierteljschr. Naturforsch. Ges. Zürich, 88:133-142, 1943.

[10] I. T. Jakobsen. A homomorphism theorem with an application to the conjecture of Hadwiger. Studia Sci. Math. Hungar., 6:151-160, 1971.

[11] T. R. Jensen and B. Toft. Graph Coloring Problems. Wiley-Interscience Series in Discrete Mathematics and Optimization. John Wiley \& Sons Inc., New York, 1995.

[12] K. Kawarabayashi and B. Toft. Any 7 -chromatic graph has $K_{7}$ or $K_{4,4}$ as a minor. Combinatorica, 25(3):327-353, 2005.

[13] A. V. Kostochka and M. Stiebitz. Partitions and edge colourings of multigraphs. Electron. J. Combin., 15(1):Note 25, 4, 2008. 
[14] U. Krusenstjerna-Hafstrøm and B. Toft. Some remarks on Hadwiger's conjecture and its relation to a conjecture of Lovász. In The theory and applications of graphs (Kalamazoo, Mich., 1980), pages 449-459. Wiley, New York, 1981.

[15] W. Mader. Homomorphiesätze für Graphen. Math. Ann., 178:154-168, 1968.

[16] N.N. Mozhan. On doubly critical graphs with the chromatic number five. Metody Diskretn. Anal., 46:50-59, 1987.

[17] V. Neumann Lara. The dichromatic number of a digraph. J. Combin. Theory Ser. $B, 33(3): 265-270,1982$.

[18] G. Royle. Gordon Royle's Small Graphs. 25-06, 2008. URL http://people.csse.uwa.edu.au/gordon/remote/graphs/index.html.

[19] M. Stiebitz. On $k$-critical $n$-chromatic graphs. In Combinatorics (Eger, 1987), volume 52 of Colloq. Math. Soc. János Bolyai, pages 509-514. North-Holland, Amsterdam, 1988.

[20] M. Stiebitz. $K_{5}$ is the only double-critical 5-chromatic graph. Discrete Math., 64(1): 91-93, 1987.

[21] B. Toft. Colouring, stable sets and perfect graphs. In Handbook of Combinatorics, Vol. 1, pages 233-288. Elsevier, Amsterdam, 1995.

[22] B. Toft. A survey of Hadwiger's conjecture. Congr. Numer., 115:249-283, 1996. 